\title{
Dynamic nuclear polarization with an inhomogeneously broadened ESR line. I. Theory
}

\author{
David S. Wollan* \\ Physics Department, Virginia Polytechnic Institute and State University, Blacksburg, Virginia 24061
}

(Received 30 May 1974; revised manuscript received 30 October 1975)

\begin{abstract}
Theoretical investigations of the dynamic nuclear polarization (DNP) and the nuclear spin-lattice relaxation time $T_{n}$ in diamagnetic dielectric crystals diluted with electron paramagnetic impurities are reported here. The steady-state DNP enhancement $E_{s s}$ and the DNP pump time $\tau_{\mathrm{DNP}}$ are calculated for the solid effect (SE), the cross effect (CE), and DNP by the electron dipole-dipole reservoir (EDDR), assuming (i) a predominantly inhomogeneously broadened (IHB) ESR line with uniform spin-packet widths (for SE and CE, but not EDDR DNP), (ii) rapid nuclear spin diffusion, (iii) the electron and nuclear high-temperature limit, (iv) no phonon bottleneck, and ( $v$ ) isotropic electron impurities or axially symmetric electrons whose symmetry axis ( $c$ axis) is aligned parallel to the applied magnetic field $\overrightarrow{\mathbf{H}}_{0}$. DNP results for IHB SE, which include DNP leakage factors, predict $E_{\mathrm{ss}}$ comparable to the ideal enhancement in favorable cases, with $E_{\mathrm{ss}}$ vs $H_{0}$ curves not proportional to the ESR line-shape derivative at high microwave powers, contrary to early theories of IHB $\mathrm{SE}$. The effects of electron jumps between spin packets are discussed. DNP and $T_{n}$ are calculated for the cross effect for both the well-resolved and unresolved limits. We get larger SE DNP and smaller CE DNP for the unresolved CE than do Hwang and Hill, and consider CE DNP leakage factors they omitted. The inequality $T_{n} / 2 \leq \tau_{\mathrm{DNP}} \leq T_{n}$ is found for the well-resolved CE, while $\tau_{\mathrm{DNP}} \approx T_{n}$ is estimated for the unresolved CE, different from the general SE case where $\tau_{\mathrm{DNP}}<T_{n}$ often occurs. DNP by EDDR is extended from the pure homogeneously broadened (HB) ESR limit to the IHB case with fast spectral diffusion, as suggested by Abragam and Borghini, and is further extended from electronic isotropy to axially symmetric electrons with the $c$ axis parallel to $\overrightarrow{\mathrm{H}}_{0}$. Various limits of $E_{\mathrm{ss}}$ and $\tau_{\mathrm{DNP}}$ are compared and contrasted for SE, CE, and EDDR DNP, to enable experimenters to differentiate these three DNP mechanisms, if possible. We propose a three-spectralregion model for wide ESR lines, which may exhibit IHB behavior in the wings of the line, HB EDDR effects near the ESR absoption peak, and CE effects in between.
\end{abstract}

\section{INTRODUCTION}

Theoretical and experimental work on dynamic nuclear polarization ${ }^{1-8}$ (DNP) with an inhomogeneously broadened ${ }^{9}$ (IHB) ESR line is reported here and in the following paper ${ }^{10}$ (called II, hereafter). Early theories ${ }^{1,2,11,12}$ of DNP for the case of IHB ESR lines with unresolved satellites, which were based implicitly on a strict shell-of-influence model for the nuclei, predicted DNP vs magnetic field curves proportional to the ESR absorption derivative $d G / d H$, with DNP enhancements reduced by one or more orders of magnitude from the ideal enhancement $E_{i}$. Khutsishvili ${ }^{13}$ and Zegers and van Steenwinkel ${ }^{14}$ pointed out the two additional mechanisms of nuclear-spin diffusion and of electron jumps between spin packets. ${ }^{15}$ They predicted DNP curves not proportional to $d G / d H$ at high microwave powers, with enhancements comparable to $E_{i}$ in favorable cases. In addition, Zegers and van Steenwinke ${ }^{14}$ gave experimental results on the DNP of ${ }^{19} \mathrm{~F}$ nuclei in LiF with $V_{K}$ centers in rough agreement with theory.

In previous work ${ }^{16,17}$ we have extended the theory of Khutsishvili ${ }^{13}$ and Zegers and van Steenwinke ${ }^{14}$ to include the DNP leakage factor which they omitted, and reported experiments on the DNP of protons in single crystals of erbium-doped yttrium ethyl sulphate (YES : Er) at $9.1 \mathrm{GHz}$ and $4.15 \mathrm{~K}$. A more comprehensive theoretical treatment is given in this paper; our experimental results will be given and analyzed in II.

In Sec. II of this paper, DNP by the solid-effect $^{1-8}(\mathrm{SE})$ mechanism for IHB ESR lines is discussed. We propose a muffin-tin model for the rapid nuclear-spin-diffusion limit which gives similar, though more general, results than those in Refs. 13 and 14.

In Sec. III, we consider a competing mechanism to $\mathrm{SE}$, called the "cross effect" (CE), 18-24 which is essentially a two-electron-one-nucleus cross relaxation that exactly conserves Zeeman energy, and thus does not require the electron dipole-dipole reservoir (EDDR). ${ }^{5,6,8}$ Following the idea of Borghini, ${ }^{24}$ we first calculate the well-resolved CE, in which there are two narrow ESR lines separated by exactly one nuclear Larmor frequency. This is then extended to a single IHB ESR line with unresolved satellites, using different approximations from those of Hwang and Hill, ${ }^{21-23}$ so that results different from theirs are obtained. Specific ways of distinguishing SE from CE are suggested.

Possible effects of EDDR on the DNP and nuclear relaxation are discussed in Sec. IV. DNP with the fast spectral-diffusion model ${ }^{6,24}$ is summarized and compared with DNP by SE and CE. We give results for EDDR effects for an axially symmetric paramagnetic center with the applied magnetic field $\overrightarrow{\mathrm{H}}_{0}$ parallel to the crystal symmetry axis. (Previous EDDR calculations $\mathrm{s}^{5,6,8,24,25}$ assume elec- 
tronic isotropy.) These results are used in Sec. $\mathrm{V}$ to propose a three-spectral-region model for wide ESR lines, which may exhibit IHB behavior in the wings of the line, homogeneous broadening 9,26 (HB) EDDR effects near the ESR absorption peak, and $C E$ effects in between. Finally, the summary is given in Sec. VI.

\section{SOLID-EFFECT DNP WITH IHB ESR LINES}

\section{A. Introduction}

We consider a system of $N_{n}$ spin $-\frac{1}{2}$ nuclei per $\mathrm{cm}^{3}$ in an insulating diamagnetic solid which is weakly doped with $N_{e}$ paramagnetic impurities (electrons, hereafter) per $\mathrm{cm}^{3}$ with effective spin $\frac{1}{2}$. In the experiments reported in II, the host lattice yttrium ethyl sulphate, $\mathrm{Y}\left(\mathrm{C}_{2} \mathrm{H}_{5} \mathrm{SO}_{4}\right)_{3} \cdot 9 \mathrm{H}_{2} \mathrm{O}$, which has 33 hydrogen nuclei per molecule, is doped $<1 \%$ with $\mathrm{Er}^{3+}$ ions. The electron and nuclear spins, each of gyromagnetic ratio $\gamma_{e}<0, \gamma_{n}>0$, respectively, are subject to a constant magnetic field $\overrightarrow{\mathrm{H}}_{0}$ in the $z$ direction and to a small ESR $\mathrm{rf}$ field of amplitude $2 H_{1}$ oscillating in the $x$ direction at a variable angular frequency $\omega_{\text {。 }}$ The electron Larmor frequency $\omega_{e}$ is defined by $\omega_{e} \equiv\left|\gamma_{e} H_{0}\right|$, the nuclear Larmor frequency $\omega_{n}$ by $\omega_{n} \equiv\left|\gamma_{n} H_{0}\right|$. This sign convention for $\omega_{e}$ and $\omega_{n}$ is different from Byvik and Wollan ${ }^{8}$ (denoted BW, hereafter) and from Sec. IV and Appendix A of this paper, but will be used elsewhere in this paper and in II. We assume $\gamma_{e}$ to be isotropic until further notice. We use the relation $g_{e} \mu_{B}=\left|\gamma_{e}\right| \hbar$, where $g_{e}$ is the electron $g$ factor, $\mu_{B}$ is the Bohr magneton, and $\hbar$ is Planck's constant over $2 \pi$.

For use below, we consider first the case of a homogeneously broadened ESR line whose width $\Delta \omega$ satisfies $\Delta \omega \ll \omega_{n}$ (well-resolved case). Ignoring EDDR effects, ${ }^{8}$ Jeffries et al. ${ }^{1-5}$ and Borghini ${ }^{7}$ describe such a system by a rate-equation approach in the laboratory frame. They show

$$
\begin{aligned}
& \frac{d P(t)}{d t}=-\frac{P(t)-P^{e}}{T_{e}}-W^{0} P(t)-\frac{N_{n}}{N_{e}} W^{ \pm}[P(t) \mp p(t)] \\
& \frac{d p(t)}{d t}=-\frac{p(t)-p^{e}}{T_{n}}-W^{ \pm}[p(t) \mp P(t)]
\end{aligned}
$$

where at most one of the three transitions $W^{0}$, $W^{ \pm}$can be excited at a given $\omega$ for fixed $H_{0}$. The electronic polarization $P(t)$ and nuclear polarization $p(t)$ are defined in Refs. 1-5. The thermalequilibrium polarizations are $P^{e}=-\tanh \left(\hbar \omega_{e} / 2 k T\right)$ and $p^{e}=\tanh \left(\hbar \omega_{n} / 2 k T\right)$, where $k$ is the Boltzmann constant and $T$ is the absolute temperature. We define the ideal enhancement $E_{i} \equiv-P^{e} / p^{e}>0$ 。 The electron and nuclear spin-lattice relaxation times are denoted $T_{e}$ and $T_{n}$. We may write ${ }^{4,5,7,8}$

$$
1 / T_{n}=1 / T_{n}^{Z}+1 / T_{n}^{L},
$$

where $1 / T_{n}^{Z}$ is the nuclear relaxation rate due to the desired paramagnetic impurity (e.g., Er in YES :Er). The nuclear leakage relaxation rate $1 / T_{n}^{L}$ is caused by undesired impurities and extraneous modes of relaxation, but explicitly excludes CE nuclear relaxation (Sec.III) and EDDR nuclear relaxation (Sec。IV).

The main ESR transition probability $W^{0}$ peaks at $\omega=\omega_{e}$ with the usual result $W^{0}=\pi \omega_{1}^{2} g(\omega)$, where $\omega_{1}^{2} \equiv\left(\gamma_{e} H_{1}\right)^{2}$ and $g(\omega)$ is the ESR line-shape function normalized to unity. The two satellite transitions $W^{ \pm}=\pi\left(\epsilon^{2} / \omega_{n}^{2}\right) \omega_{1}^{2} g\left(\omega \pm \omega_{n}\right)$ peak at $\omega=\omega_{e} \pm \omega_{n}$, and are usually weaker than $W^{0}$ since $\epsilon^{8} / \omega_{n}^{2}$ is ordinarily $\ll 1$. This calculation is valid in the rapid nuclear-spin-diffusion limit ${ }^{3-8,13,27-30}$ for an HB line with well-resolved satellites, an isotropic $g_{e}$, no phonon bottleneck, ${ }^{2,4,5,7}$ and no EDDR effects. $5,6,8$ Throughout this paper we assume (a) no phonon bottleneck and (b) the electron (and thus nuclear) high-temperature limit, $\hbar \omega_{e} / k T \ll 1$. Corrections for DNP and $T_{n}$ at low temperatures have been given previously. ${ }^{3-5,7}$ Underlying Eqs. (1) and (2) is the idea that the sample is broken up into $N_{e}$ shells of influence of outer radius $R$ $\equiv\left(3 / 4 \pi N_{e}\right)^{1 / 3}$. The inner radius is the diffusionbarrier radius $d .^{3-8}$ For dilute systems each nucleus interacts most strongly with the electron at the center of its shell. In an HB line, each shell is equivalent to every other. Hence, an average over a single shell of one electron and $N_{n} / N_{e}$ nuclei is representative of the whole sample.

Consider now saturation of the $W^{ \pm}$satellite $\left(W^{0}=0=W^{\mp}\right)$. In the limit $T_{e} \ll T_{n}, N_{e} \ll N_{n}, P(t)$ comes to quasiequilibrium $(d P / d t \approx 0)$ in times $t \ll T_{n}$. We may solve Eq. (1) for $P(t)$, and substitute into Eq. (2), using the DNP enhancement $E(t) \equiv p(t) / p^{e}$. Eq. (2) then becomes

$$
\frac{d E(t)}{d t}=-\frac{1}{\tau_{\mathrm{DNP}}}\left[E(t)-E_{\mathrm{ss}}\right],
$$

where the steady-state enhancement $E_{\text {ss }}$ is

$$
E_{\mathrm{ss}}=\frac{\left(1+f_{e} S^{ \pm}\right) \mp E_{i} S^{ \pm}}{1+S^{ \pm}\left(1+f_{e}\right)}
$$

and the DNP pump time $\tau_{\mathrm{DNP}}$ is

$$
\frac{1}{\tau_{\mathrm{DNP}}}=\frac{1}{T_{n}}\left[\frac{1+S^{ \pm}\left(1+f_{e}\right)}{1+f_{e} S^{ \pm}}\right] \text {. }
$$

Equations (4) and (6) are valid for coarse-grained times $t$ such that $T_{e} \ll t \ll \tau_{\text {DNP. }}$. We use the DNP leakage factor $f_{e} \equiv N_{n} T_{e} / N_{e} T_{n}$ and the satellite saturation parameter $S^{ \pm} \equiv W^{ \pm} T_{n}$. DNP by the $W^{ \pm}$ transitions alone is called the solid effect. ${ }^{1-8}$

\section{B. Strict shell-of-influence model}

We now consider the case of an ESR line with unresolved SE satellites whose IHB mechanisms 


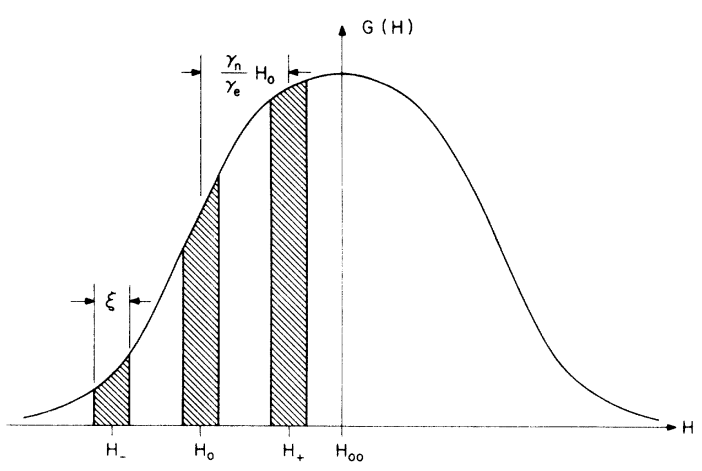

FIG. 1. Inhomogeneously broadened (IHB) ESR lineshape function $G(H)$.

completely dominate those due to $\mathrm{HB}^{9}{ }^{9}$ The ESR line-shape envelope function $G(H)$, normalized to unity, can then be considered to be composed of a large number of independent HB spin packets, as in Fig. 1. For fixed $\omega$, the $W^{0}$ transition occurs at the variable applied magnetic field $H_{0}$, with the ESR line center located at $H_{00}=\hbar \omega / g_{e} \mu_{B}$. The two satellite transitions $W^{ \pm}$are excited simultaneously at ${ }^{13} H_{ \pm}=H_{0}\left[1 \pm\left(\omega_{n} / \omega_{e}\right)\right]$.

We assume the physically unreasonable rectangular spin packets of width $\xi$ in Fig。 1 for ease of the DNP analysis. Alternatively, it may be possible to use more physical spin-packet shapes, provided that the wings of the packets fall off sufficiently rapidly to insure negligible cross relaxation between packets. In this case, $\xi$ may be treated as an effective width such that $[\xi G(H)] N_{e}$ is the number of electrons per $\mathrm{cm}^{3}$ in the spin packet centered at $H$.

The width $\xi$ in Fig. 1 has been taken $\mathrm{as}^{1,2}$

$$
\xi \approx\left(H_{\text {in }}^{2}+H_{1}^{2}+H_{m}^{2}\right)^{1 / 2},
$$

where $H_{\text {in }}$ is the intrinsic HB width and $H_{m}$ is the amplitude of the magnetic field modulation applied at frequency $f_{m}$. Equation (7) is valid if the condition $f_{m} \gtrsim\left(T_{e}\right)^{-1}, \omega$ holds. However, if $f_{m} \ll\left(T_{e}\right)^{-1}$, $\omega$, the magnetic field modulation is not an $\mathrm{HB}$ mechanism, and we must use

$$
\xi \approx\left(H_{\text {in }}^{2}+H_{1}^{2}\right)^{1 / 2} .
$$

In this latter case, and assuming $H_{m}>H_{1 \mathrm{n}}, H_{1}$, the field modulation sweeps slowly back and forth through a series of spin packets each of which comes to internal equilibrium in times $t \ll\left(f_{m}\right)^{-1}$. Evidence for this is given in $\Pi$. Throughout this paper, we assume the condition $\xi<\left|\gamma_{n} / \gamma_{e}\right| H_{0}$.

In what we call the strict shell-of-influence model, the nuclei in a given shell are influenced only by their own electron, which is assumed fixed in a given spin packet in time; nuclear-spin diffusion between shells is assumed negligible. ${ }^{1,2,11,12}$
If $\xi$ is determined only by $H_{1}, H_{m}$, and $T_{e}$ processes (and not by electron spin-spin relaxation time $T_{2 e}$ processes), $\xi$ is not a function of $G(H)$. We may then use the equations for well-resolved satellite DNP for each independent spin packet, but weight the nuclei by $\xi G(H)$. Thus, from Eq. (5), the sample average steady-state enhancement is

$$
\begin{aligned}
E_{\mathrm{ss}}= & \left\{1-\xi\left[G\left(H_{+}\right)+G\left(H_{-}\right)\right]\right\}(1) \\
& +\left[\xi G\left(H_{-}\right)\right] \frac{\left(1+f_{e} S^{-}\right)+E_{i} S^{-}}{1+S^{-}\left(1+f_{e}\right)} \\
& +\left[\xi G\left(H_{+}\right)\right] \frac{\left(1+f_{e} S^{+}\right)-E_{i} S^{+}}{1+S^{+}\left(1+f_{e}\right)} .
\end{aligned}
$$

If $E_{i} \gg 1, E q .(9)$ becomes

$$
E_{\mathrm{ss}}=1+\frac{E_{i} S \xi\left[G\left(H_{-}\right)-G\left(H_{+}\right)\right]}{1+S\left(1+f_{e}\right)},
$$

where $S=S^{ \pm}$holds when $\xi$ is determined by $H_{1}$, $H_{m}$, and $T_{e}$ alone. Thus $E_{\mathrm{ss}}$ is proportional to the absolute difference $\xi\left[G\left(H_{-}\right)-G\left(H_{+}\right)\right]$of the spinpacket populations。In the typical limit $\xi \ll \mid H_{ \pm}$ $-H_{0} \mid \ll\left[G\left(H_{00}\right)\right]^{-1}$, Eq. (10) becomes (by a Taylorseries expansion) $E_{\mathrm{ss}} \propto d G / d H$ at microwave powers $S \gtrsim 1$. The peak values of $E_{\mathrm{ss}}$ at $S \gg 1, f_{e} \ll 1$, are smaller than $E_{i}$ by one or more orders of magnitude. A schematic diagram of this case is shown in Fig. 2, where most of the nuclei are in shells with $E_{\mathrm{ss}}=1$, while those few nuclei in the shells of electrons in the $G\left(H_{ \pm}\right)$spin packets are polarized to $E_{\mathrm{ss}}=\mp E_{i}$ 。

A point sometimes overlooked is that in this

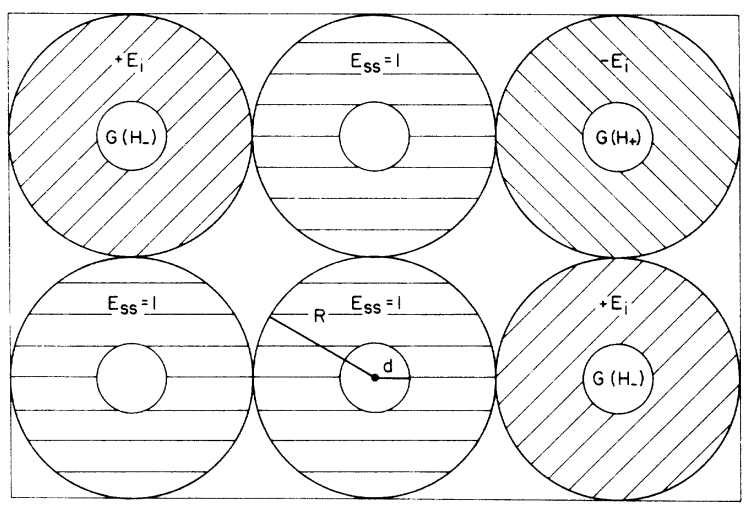

FIG. 2. Strict shell-of-influence model for DNP with an IHB ESR line. Bulk nuclei are defined to lie within shells defined by the diffusion-barrier radius $d$ and the shell outer radius $R=\left(3 / 4 \pi N_{e}\right)^{1 / 3}=0.62 N_{e}^{-1 / 3}$. For pictorial convenience only, we take $R=\frac{1}{2}\left(N_{e}\right)^{-1 / 3}$ in this figure, thus neglecting some nuclei at distances $r>R$ from any electron. Nuclei in shells whose electrons lie in the spin packets $G\left(H_{ \pm}\right)$are polarized to $E_{s}=\mp E_{i}$ at full microwave power and $f_{e} \ll 1$. The nuclei in all other shells are at $E_{\mathbf{s s}}=1$ in this model. See Eqs. (9) and (10) in the text. 
model, for times $t \gg T_{e}$, the NMR signal monitoring the DNP will in general respond with two time constants: $\tau_{\text {DNP }}$ from Eq. (6) for the nuclei in the $G\left(H_{ \pm}\right)$packets, and $T_{n}$ for all the other nuclei. It is easy to think of DNP experiments to observe this, e.g. , by changing $H_{0}$ (at fixed $\omega$ ) in times $t$ such that $T_{e} \ll t \ll \tau_{\mathrm{DNP}}$.

\section{Muffin-tin model}

The assumption of no nuclear-spin diffusion between shells of influence is not physically plausible, especially in the rapid-diffusion limit. ${ }^{4-6,13,27-30}$ Therefore, we propose a muffin-tin model (Fig. 3) for DNP by the solid effect in an IHB ESR line. For the moment we assume that each electron is fixed in a given spin packet in time.

We assume that nuclear-spin diffusion is sufficiently rapid so that all the bulk nuclei outside the diffusion barriers come to a common average nuclear polarization $\bar{p}(t)$. Thus the time evolution equations of $\bar{p}(t)$ and $P_{ \pm}(t)$ can be written from Eqs. (1)-(3), weighting the $\bar{p}(t)$ rates by the relative spin-packet populations:

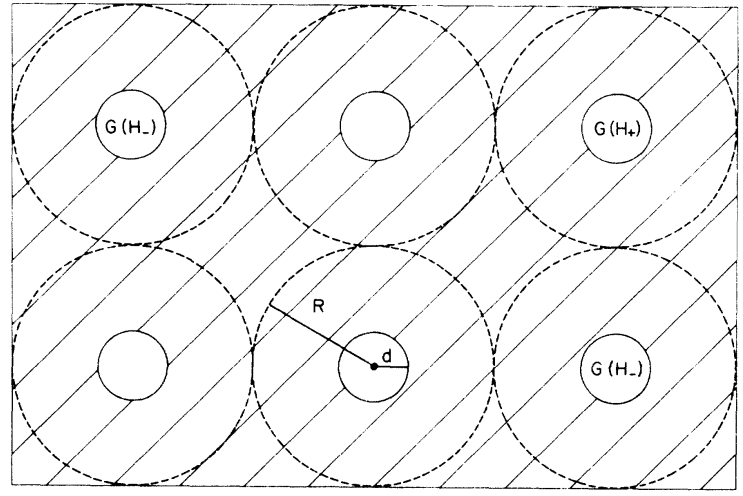

FIG. 3. Muffin-tin model for DNP with an IHB ESR line. Rapid nuclear-spin diffusion brings all the bulk nuclei to a common polarization given by Eq. (14) in the text. Bulk nuclei are all those lying outside their own diffusion-barrier radius $d$. For comparison with Fig. 2 only, we take $R=\frac{1}{2} N_{e}^{-1 / 3}$ in this figure.

$$
\frac{d P_{ \pm}(t)}{d t}=-\frac{P_{ \pm}(t)-P^{e}}{T_{e}}-\frac{N_{n}}{N_{e}} W^{ \pm}\left[P_{ \pm}(t) \mp \bar{p}(t)\right],
$$

Each independent spin packet has its own electronic polarization $P(t)$ : the two of interest for DNP are $P_{ \pm}(t)$ for the packets $G\left(H_{ \pm}\right)$. In the rapid nuclear-spin-diffusion limit, all the $\bar{p}(t)$ rate terms are linearly additive. The first term on the right-hand side of Eq. (12) is the nuclear leakage relaxation rate. The second term is nuclear relaxation by the electrons in all spin packets except $G\left(H_{ \pm}\right)$, and the last two terms are the DNP and nuclear relaxation rate contributions to $\bar{p}(t)$ from the two spin packets $G\left(H_{ \pm}\right)$.

If $T_{e} \ll T_{n}$ and $N_{n} \gg N_{e}$, the two electronic polarizations $P_{ \pm}(t)$ come to quasiequilibrium $\left(d P_{ \pm} / d t\right.$ $\approx 0$ ) in times $t \ll T_{n}$. We may solve Eq. (11) for $P_{ \pm}(t)$ and substitute into Eq. (12). Using $S=S^{ \pm}$ $=W^{ \pm} T_{n}, E(t)=\bar{p}(t) / p^{e}$, and Eq. (3), it is easy to show

$$
\frac{d E(t)}{d t}=-\frac{1}{\tau_{\mathrm{DNP}}}\left[E(t)-E_{\mathrm{ss}}\right]
$$

where

$$
E_{\mathrm{ss}}=\frac{\left(1+f_{e} S\right)+E_{i} S \xi\left[G\left(H_{-}\right)-G\left(H_{+}\right)\right]}{1+S\left\{\xi\left[G\left(H_{+}\right)+G\left(H_{-}\right)\right]+f_{e}\right\}},
$$

and

$$
\frac{1}{\tau_{\mathrm{DNP}}}=\frac{1}{T_{n}}\left(\frac{1+S\left\{\xi\left[G\left(H_{+}\right)+G\left(H_{-}\right)\right]+f_{e}\right\}}{1+f_{e} S}\right) \text {. }
$$

If $E_{i} \gg 1$, then $E_{\mathrm{ss}}$ can be written as

$$
E_{\mathrm{ss}}=1+\left(\frac{E_{\infty}}{1+f_{\text {eff }}}\right)\left(\frac{S}{S+S_{1 / 2}}\right) \text {. }
$$

The infinite power $\left(S \gg S_{1 / 2}\right)$ no DNP leakage $\left(f_{e}=0\right)$ enhancement $E_{\infty}$, given by

$$
E_{\infty}=E_{i} \frac{G\left(H_{-}\right)-G\left(H_{+}\right)}{G\left(H_{+}\right)+G\left(H_{-}\right)},
$$

is proportional to $E_{i}$ and to the relative spin-packet population difference ${ }^{31}$

$$
\left[G\left(H_{-}\right)-G\left(H_{+}\right)\right] /\left[G\left(H_{+}\right)+G\left(H_{-}\right)\right] \text {。 }
$$

We find $E_{\infty} \approx 0.8 E_{i}$ in favorable cases, and note that $E_{\infty}$ is not in general proportional to $d G / d H$. The effective DNP leakage factor $f_{\text {eff }}$, given by

$$
f_{\text {eff }}=\frac{f_{e}}{\xi\left[G\left(H_{+}\right)+G\left(H_{-}\right)\right]},
$$

is larger than $f_{e}$. The physical idea is that only $N_{e}^{\text {eff }}=\xi\left[G\left(H_{+}\right)+G\left(H_{-}\right)\right] N_{e}$ electrons are polarizing the nuclei, whereas all $N_{e}$ electrons contribute to 
the nuclear spin-lattice relaxation. Thus we find $f_{\text {eff }}=N_{n} T_{e} / N_{e}^{\text {eff }} T_{n}$. Finally, the half-power parameter is

$$
S_{1 / 2}=1 / \xi\left[G\left(H_{+}\right)+G\left(H_{-}\right)\right] \text {. }
$$

So far we have assumed that the electron in a given spatial site remains fixed in one spin packet in time. The essential idea of Khutsishvili ${ }^{13,15}$ and Zegers and van Steenwinkel ${ }^{14}$ is that the electron may jump from one spin packet to another, a process that is not necessarily the same as ESR spectral diffusion (Sec. IV). (The complicated relation between these two processes will not be considered in this paper.) They also emphasize the important role of nuclear-spin diffusion. Let us define an average electron spin-packet jump time $\tau$. Then the models discussed thus far correspond to $\tau \gg T_{n^{*}}$. If $\tau \lesssim T_{n}$, the electron in a given spatial site spends only a fraction $\xi G\left(H_{ \pm}\right)$ of its time in the $G\left(H_{ \pm}\right)$packets. In the muffin-tin model, Eqs. (11)-(19) remain valid as spatial averages even when $T_{e} \ll \tau \leqslant T_{n^{\circ}}$ It makes no difference to the bulk nuclei which electron spatial sites provide the DNP $W^{ \pm}$transitions, provided only that they are weighted by the average spinpacket populations $\xi G\left(H_{ \pm}\right)$.

(a)

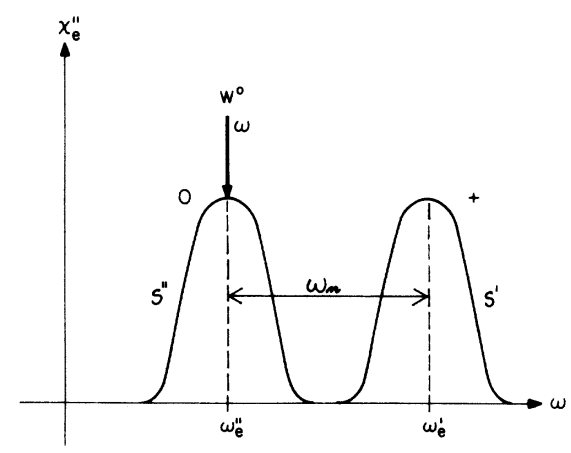

(b)

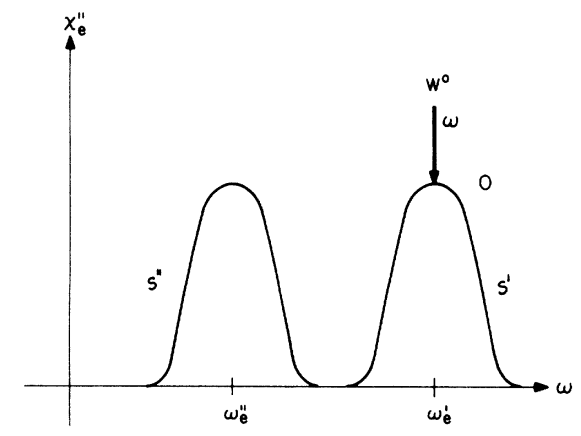

FIG. 4. ESR absorption susceptibility $\chi_{e}^{\prime \prime}$ vs $\omega$ at fixed $H_{0}$ for the well-resolved CE. Electron sites $S^{\prime}$ and $S^{\prime \prime}$ differ in their resonant frequencies by $\omega_{n}$. (a) When the allowed ESR transition $W^{0}$ is applied at $\omega=\omega_{e}^{\prime \prime}$, we denote the $S^{\prime \prime}\left(S^{\prime}\right)$ electron by $0(+)$. (b) When $W^{0}$ is applied at $\omega=\omega_{e}^{\prime}$, we denote $S^{\prime}\left(S^{\prime \prime}\right)$ by $0(-)$.
We now propose a modified shell-of-influence model defined by no nuclear-spin diffusion between shells of influence, but $\tau \leqslant T_{n^{\circ}}$ The muffin-tin model and the modified shell-of-influence model give the same DNP results for the case $T_{e} \ll \tau \ll T_{n^{\circ}}$ For the latter model in this limit, Eq. (12) may be viewed as a time average for a single shell of influence. Each shell samples many spin packets in times $t \approx T_{n^{\circ}}$ Thus, each shell is equivalent to every other, and an average over one shell is representative of the whole sample. This model is not valid if $\tau \gtrsim T_{n}$. Khutsishvili ${ }^{13,15}$ and Zegers and van Steenwinkel ${ }^{14}$ have considered DNP for the modified shell-of-influence model in the limit $T_{e}$ $\ll \tau \ll T_{n}$. However, Ref. 14 omits the $S$ and $f_{e}$ dependence of $E_{\text {ss }}$ and does not discuss $\tau_{\text {DNP. }}$. Reference 13 omits $f_{e}$ effects and gives the wrong dependence of $E_{\mathrm{ss}}$ on $T_{n}^{L}$.

The effects on IHB DNP of other time scales for electron spin-packet jumps, of various inhomogeneous broadening mechanisms, and of nuclearspin-diffusion criteria have been given elsewhere. ${ }^{32}$

\section{DNP BY THE CROSS EFFECT}

\section{A. Well-resolved cross effect}

The basic mechanism of the cross effect (CE) was first discussed by Kessenikh et al. ${ }^{18-20}$ who considered only the case of DNP by CE in the limit of large microwave power. Hwang and Hill ${ }^{21-23}$ generalized their theory to arbitrary microwave powers. Borghini ${ }^{24}$ has given an excellent review of various DNP mechanisms including $\mathrm{SE}$ and $\mathrm{CE}$, but omitted all DNP leakage factor effects. None of these workers calculated $\tau_{D N P}$. We first consider two identical narrow HB ESR lines separated by exactly one nuclear Larmor frequency $\omega_{n}$ as in Fig. 4, where the ESR linewidth $\Delta \omega$ satisfies $\Delta \omega$ $\ll \omega_{n}$. A simple model of the well-resolved cross effect has been given elsewhere. ${ }^{32}$

The basic rate equations for $\mathrm{CE}$ have been given by Hwang and $\mathrm{Hill}^{22,23}$ for the case of three electrons $S^{i}, S^{i \pm 1}$, and for a strict shell-of-influence model of the nuclei. The Larmor frequencies of electron $S^{i}, S^{i \pm 1}$ are $\omega_{e}^{i}, \omega_{e}^{i} \pm \omega_{n}$ (Fig. 5) with electron polarizations $P_{i}, P_{i \pm 1}$, respectively。The nuclear polarizations $p_{i}, p_{i \pm 1}$, refer to an average over the $N_{n} / N_{e}$ nuclei in each of the shells of influence surrounding the $S^{i}, S^{i \pm 1}$ electrons. Hwang and Hill's ${ }^{22,23}$ results (without the $W^{0}, W^{ \pm}$transitions) are

$$
\begin{aligned}
\frac{d P_{i}(t)}{d t}= & -\frac{1}{T_{e}}\left[P_{i}(t)-P_{i}^{e}\right] \\
& +\frac{N_{n}}{N_{e}} W_{i, i-1}\left[2 P_{i-1}-2 P_{i}-\left(p_{i}+p_{i-1}\right)\right] \\
& +\frac{N_{n}}{N_{e}} W_{i, i+1}\left[2 P_{i+1}-2 P_{i}+\left(p_{i}+p_{i+1}\right)\right],
\end{aligned}
$$




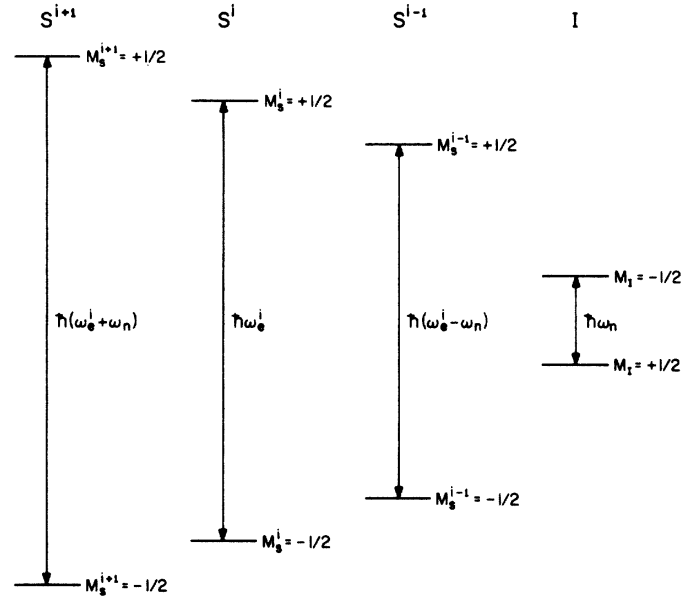

FIG. 5. Energy-level diagram of three electrons $S^{i}$, $S^{i \neq 1}$ and one nucleus $I$ at fixed $H_{0}$ for the well-resolved CE. This is adapted from Fig. 1 in Ref. 22 and Fig. 37 in Ref. 23.

$$
\begin{aligned}
\frac{d p_{i}(t)}{d t}= & -\frac{1}{T_{n}^{\prime}}\left[p_{i}(t)-p^{e}\right]+W_{i, i=1}\left(P_{i-1}-P_{i}-p_{i}\right) \\
& -W_{i, i+1}\left(P_{i+1}-P_{i}+p_{i}\right)
\end{aligned}
$$

where $T_{n}^{\prime}$ refers to all modes of nuclear spin-lattice relaxation except CE. The $W_{i, i \pm 1}$ terms (see below) are each averaged over one shell of influence with rapid diffusion assumed for each shell. We use $P_{i}^{e}=-\tanh \left(\hbar \omega_{e}^{i} / 2 k T\right)$ and $P_{i \pm 1}^{e}$ $=-\tanh \left[\hbar\left(\omega_{e}^{i} \pm \omega_{n}\right) / 2 k T\right]$.

We now specialize these equations to the case of two well-resolved ESR lines separated by $\omega_{n}$ as in Fig. 4. For example, in a crystal with two inequivalent magnetic sites per unit cell, there may be some orientation of the crystal axes relative to $\overrightarrow{\mathrm{H}}_{0}$ where this condition is fulfilled. Let there be equal numbers $\left(\frac{1}{2} N_{e}\right.$ per $\left.\mathrm{cm}^{3}\right)$ of electrons in each of the two sites $S^{\prime}, S^{\prime \prime}$. Thus we may break the crystal up into $N_{e}$ uniform shells of influence, each containing one electron and $N_{n} / N_{e}$ nuclei, and further assume that the shells of $S^{\prime}, S^{\prime \prime}$ alternate. A sample average is then obtained from an average over one pair of shells $S^{\prime}, S^{\prime \prime}$.

Consider now $\mathrm{rf}$ irradiation at applied frequency $\omega=\omega_{e}^{\prime}$ (or $\left.\omega_{e}^{\prime \prime}\right)$ with $H_{0}$ fixed, which will stimulate the allowed ESR $W^{0}$ transition for electron $S^{\prime}$ (or $S^{\prime \prime}$ ) and the forbidden $W^{+}$(or $W^{-}$) SE transition for the other electron. We shall denote the electron (and the nuclei in its shell) at $\omega$ by the subscript 0 , and the other electron and its associated nuclei by the subscript \pm , as in Fig. 4. The CE transitions $W_{i, i \pm 1}$ become $W_{0,+}, W_{0,-}$, where by symmetry $W_{0,+}=W_{0,-} \equiv W_{\mathrm{CE}} \cdot \mathrm{Hill}^{23}$ has shown

$$
W_{\mathrm{CE}}=\left(\epsilon^{2} / \omega_{n}^{2}\right)\left(T_{2 e}\right)^{-1},
$$

where $\epsilon^{2} / \omega_{n}^{2}$ is the usual satellite mixing term. ${ }^{1-8,13}$ Equation (22), valid when $\left(\omega_{n} T_{2 e}\right)^{2} \gg 1$, may be rewritten ${ }^{8}$

$$
W_{\mathrm{CE}}=\frac{3}{10}\left(\frac{g_{e} \mu_{B}}{H_{0}}\right)^{2} \frac{1}{d^{3} R^{3}} \frac{1}{T_{2 e}^{B}},
$$

where $T_{2 e}$ in Eq. (22) is replaced by an appropriate $T_{2 e}^{B}$ for the reasons given in Appendix A. The mechanism of forbidden (or satellite) electron spin-spin transitions $\left(\Delta M_{S}^{\prime}= \pm 1, \Delta M_{S}^{\prime \prime}=\mp 1, \Delta M_{I}\right.$ $= \pm 1$ ) causes both CE here and EDDR DNP and nuclear relaxation (Sec. IV). Electron and nuclear Zeeman energy are exactly conserved in CE but not in EDDR theory; in the latter it is precisely the role of the EDDR to absorb (or emit) the excess (or deficit) of Zeeman energy. ${ }^{6,8}$

We now write equations for the electron polarizations $P_{0}(t), P_{t}(t)$, and the nuclear polarization $p(t)$ for two neighboring shells of influence, using Eqs. (20)-(23) and adding the $W^{0}$ transition. It is easy to show from Eq. (21) that $p_{0}(t)=p_{ \pm}(t)$. Hence we define

$$
p(t) \equiv p_{0}(t)=p_{ \pm}(t) .
$$

The rate equation results then become

$$
\begin{aligned}
\frac{d P_{0}(t)}{d t}= & -\frac{1}{T_{e}}\left[P_{0}(t)-P_{0}^{e}\right]-W^{0} P_{0}(t) \\
& +\frac{N_{n}}{N_{e}}\left(2 W_{\mathrm{CE}}\right)\left[P_{ \pm}(t)-P_{0}(t)_{ \pm} p(t)\right], \\
\frac{d P_{ \pm}(t)}{d t}= & -\frac{1}{T_{e}}\left[P_{ \pm}(t)-P_{ \pm}^{e}\right] \\
& +\frac{N_{n}}{N_{e}}\left(2 W_{\mathrm{CE}}\right)\left[P_{0}(t)-P_{ \pm}(t) \mp p(t)\right], \\
\frac{d p(t)}{d t}= & -\frac{1}{T_{n}^{\prime}}\left[p(t)-p^{e}\right] \\
& -W_{\mathrm{CE}}\left\{p(t) \mp\left[P_{0}(t)-P_{ \pm}(t)\right]\right\} .
\end{aligned}
$$

We may solve Eqs. (25)-(27) in the manner of Sec. II to get

$$
\frac{d p(t)}{d t}=-\frac{1}{\tau_{\mathrm{DNP}}}\left[p(t)-p_{\mathrm{ss}}\right]
$$

where

$$
p_{\mathrm{ss}}=p^{e} \mp P_{0}^{e}\left(\frac{S_{\mathrm{CE}}}{1+S_{\mathrm{CE}}\left(1+f_{e}^{\prime}\right)}\right)\left(\frac{S^{0}}{S^{0}+S_{1 / 2}^{\mathrm{CE}}}\right),
$$

and

$$
\frac{1}{\tau_{\mathrm{DNP}}}=\frac{1}{T_{n}^{\prime}}+\frac{W_{\mathrm{CE}}\left(1+S^{0}\right)}{\left(1+S^{0}\right)\left(1+f_{e}^{\prime} S_{\mathrm{CE}}\right)+f_{e}^{\prime} S_{\mathrm{CE}}} .
$$

We have used $S^{0}=W^{0} T_{e}, S_{\mathrm{CE}}=W_{\mathrm{CE}} T_{n}^{\prime}, f_{e}^{\prime}=2 N_{n} T_{e} /$ $N_{e} T_{n}^{\prime}, P_{ \pm}^{e}=P_{0}^{e} \mp p^{e}$, and

$$
S_{1 / 2}^{\mathrm{CE}}=\frac{1+S_{\mathrm{CE}}\left(1+2 f_{e}^{\prime}\right)}{1+S_{\mathrm{CE}}\left(1+f_{e}^{\prime}\right)} .
$$

We note that $1 \leq S_{1 / 2}^{\mathrm{CE}} \leq 2$. 
Let us consider first nuclear spin-lattice relaxation with Eqs. (28)-(31), with $S^{0}=0$ and no $\mathrm{DNP}$. We see that $p_{\mathrm{ss}}$ reduces to $p^{e}$, and $\tau_{\mathrm{DNP}}$ reduces to $T_{n}$ where

$$
\frac{1}{T_{n}}=\frac{1}{T_{n}^{\prime}}+\frac{1}{T_{n}^{C E}}
$$

and

$$
\frac{1}{T_{n}^{\mathrm{CE}}}=\frac{W_{\mathrm{CE}}}{1+2 f_{e}^{\prime} S_{\mathrm{CE}}}
$$

Now we consider DNP by CE. When $S^{0}, S_{\mathrm{CE}}$ $\gg 1$, Eq. (29) predicts $p_{\mathrm{ss}}=\mp P_{0}^{e} /\left(1+f_{e}^{\prime}\right)$, which gives large DNP which can be reduced only by a significant DNP leakage factor $f_{e}^{\prime} \gtrsim 1$. Note first that $f_{e}^{\prime}$ depends only on the non-CE nuclear relaxation time $T_{n}^{\prime}$ and not on $T_{n}^{\mathrm{CE}}$. The factor 2 in $f_{e}^{\prime}$ comes in because one electron (e.g.,$\left.S^{\prime}\right)$ is pumped by $W^{0}$, while the other electron (e.g. , $S^{\prime \prime}$ ) relaxes to the lattice (see, e.g., Fig. 4 in Ref. 24). For two adjacent shells of influence $\left(S^{\prime}\right.$ and $\left.S^{\prime \prime}\right)$, one electron $S^{\prime \prime}$ relaxing at a rate $1 / T_{e}$ is servicing $2 N_{n} / N_{e}$ nuclei, and the CE DNP is reduced when $\left(2 N_{n} / N_{e}\right) / T_{n}^{\prime} \gtrsim 1 / T_{e}$. Second, since $W_{\mathrm{CE}}$ depends on $T_{2 e}$, which in turn depends on $N_{e}$, we see that $S_{\mathrm{CE}}$ will in general vary as $N_{e}$ varies. However, there may be a range of variation of $N_{e}$ such that $S_{\mathrm{CE}}$ remains $\gg 1$, in which case $E_{\mathrm{ss}}=p_{\mathrm{ss}} / p^{e}$ will be independent of $N_{e}$. Third, $E_{s s}$ can be reduced either by low microwave power $\left(S^{0} \leqslant 1\right)$ or by a low CE saturation parameter $S_{\mathrm{CE}} \lesssim 1$. These results may be compared with the well-resolved SE DNP in Eq. (5).

Using Eqs. (30) and (32), it is easy to show that $\tau_{\text {DNP }}$ can be written

$\frac{1}{\tau_{\mathrm{DNP}}}=\left(\frac{1}{T_{n}}\right) \frac{1+S^{0}\left\{\left[1+S_{\mathrm{CE}}\left(1+f_{e}^{\prime}\right)\right] /\left[1+S_{\mathrm{CE}}\left(1+2 f_{e}^{\prime}\right)\right]\right\}}{1+S^{0}\left[\left(1+f_{e}^{\prime} S_{\mathrm{CE}}\right) /\left(1+2 f_{e}^{\prime} S_{\mathrm{CE}}\right)\right]}$.

We see that $\tau_{\mathrm{DNP}}=T_{n}$ if $f_{e}^{\prime} S_{\mathrm{CE}}, f_{e}^{\prime} \ll 1$ or if $S^{0} \ll 1$. We find in general

$$
\frac{1}{2} T_{n} \leq \tau_{\mathrm{DNP}} \leq T_{n} \text {. }
$$

This limited range of $\tau_{D N P}$ for the well-resolved CE is to be contrasted in SE with $\tau_{\mathrm{DNP}} \approx f_{e} T_{n} \ll T_{n}$ from Eq. (6) with $S^{ \pm} \gg 1, f_{e} \ll 1$.

Although Hwang and $\mathrm{Hill}^{22,23}$ did not work out the well-resolved $C E$, their unresolved $C E$ results reduce to essentially our equations in the appropriate limit except in one respect. They applied detailed balance to the $W_{\mathrm{CE}}$ transitions in Eqs. (20), (25), and (26), obtaining a complete cancellation of the CE terms in the electron polarization equations. This involves adding $d P_{0} /$ $d t$ and $d P_{t} / d t$, which is meaningful only if $P_{0}=P_{t}$, which contradicts an essential hypothesis of CE. The consequence is that they get no DNP leakage factors $f_{e}^{\prime}$ in $p_{\text {ss }}$. The "leakage factor" $\beta$ in Refs.
22 and 23 is essentially $\beta \approx 1 / S_{C E}$. We have treated $S_{\mathrm{CE}}=W_{\mathrm{CE}} T_{n}^{\prime}$ as a CE saturation or effectiveness parameter here. Their use of $\beta$ leakage is an alternative and correct approach to our use of $S_{\mathrm{CE}}$. However, CE DNP leakage in this paper will always refer to $f_{e}^{\prime}$ and never to $\beta$, and must be included in a correct treatment of CE DNP.

It is easy in principle to add the $W^{ \pm} \mathrm{SE}$ transitions to Eqs. (26) and (27) in order to show the combined effect of SE and CE. However, the algebra gets messy when $f_{e}, f_{e}^{\prime} \gtrsim 1$, so we have obtained no results for this general case. Borghini ${ }^{24}$ has given the result for $f_{e}, f_{e}^{\prime} \ll 1$.

\section{B. Unresolved cross effect with an IHB ESR line}

We now investigate CE for a single IHB ESR line of width $\Delta \omega$ with unresolved satellites $\left(\Delta \omega \gtrsim \omega_{n}\right)$, as in Fig. 6, the case for which CE has been analyzed in detail previously. ${ }^{18-23}$ [Parenthetically, we note that no CE is possible for a single ESR line with well-resolved satellites $\left(\Delta \omega \ll \omega_{n}\right)$, since no two electrons in the sample will differ in their Larmor frequencies by $\omega_{n^{*}}$ ] An ESR line with unresolved satellites must be IHB in order to have $\mathrm{CE}$, since each spin packet, e。g。, $G\left(H_{0}\right), G\left(H_{ \pm}\right)$, $G\left(H_{ \pm \pm}\right)$, in Fig。6, must have its own well-defined electron polarization. If spectral diffusion (Sec. IV) brings the spin packets into common mutual equilibrium in times $\tau \ll T_{e}$, then CE is wiped out. ${ }^{23,24}$

Since all the electron polarizations $P_{i}$ in Eqs. (20) and (21) are coupled to one another by the $W_{i, i \pm 1}$ terms, a closed solution of those equations is not possible. Hence, we shall ignore the $W_{i, i \neq 1}$ terms in Eq. (20) but not in Eq. (21). This amounts to ignoring the $f_{e}^{\prime}, f_{e}^{\prime} S_{\mathrm{CE}}$ leakage factors in $T_{n}^{\mathrm{CE}}$ and DNP. Although these are not in principle ignorable, we do so for computational convenience, and then extrapolate the results of Sec. III A to estimate their probable effect here.

The electron polarization equations for each of

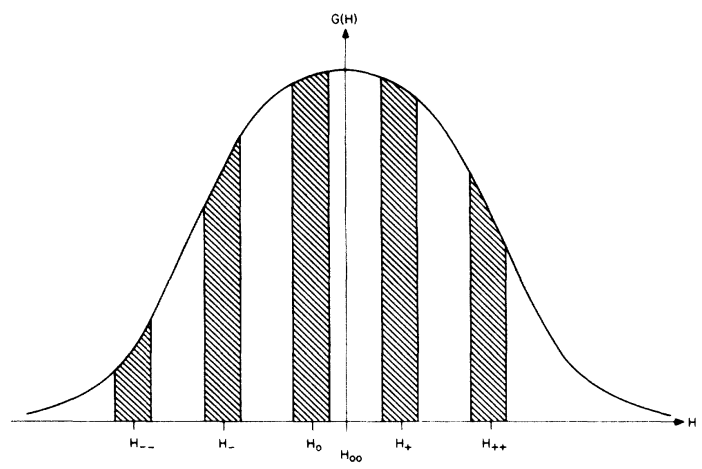

FIG. 6. IHB ESR line-shape function $G(H)$ with five spin packets $H_{0}, H_{ \pm}, H_{ \pm \pm}$. 
the $N$ spin packets in the ESR line may now be written as

$$
\begin{aligned}
& \frac{d P_{0}(t)}{d t}=-W^{0} P_{0}(t)-\frac{1}{T_{e}}\left[P_{0}(t)-P_{0}^{e}\right], \\
& P_{ \pm}(t)=P_{0}^{e} \pm p^{e}, \\
& P_{ \pm \pm}(t)=P_{0}^{e} \pm 2 p^{e}, \\
& P_{i}(t)=P_{i}^{e}, \\
& P_{i \pm 1}(t)=P_{i \pm p^{e}}^{e} .
\end{aligned}
$$

The subscripts $0, \pm, \pm \pm$, refer to the spin packets at $H_{0}, H_{ \pm}=H_{0}\left(1 \pm\left|\gamma_{n} / \gamma_{e}\right|\right)$, and $H_{ \pm \pm}=H_{0}\left(1 \pm 2\left|\gamma_{n} / \gamma_{e}\right|\right)$, respectively, while $i$ refers to all other spin pack- ets. By omitting the $W_{i, i \pm 1}$ terms in Eq. (20), we assume that all the electron polarizations except $P_{0}(t)$ are at their thermal-equilibrium values in times $t \gg T_{e}$. On the same time scale, $P_{0}(t)$ attains quasiequilibrium $\left(d P_{0} / d t \approx 0\right)$, so that Eq. (35a) may be solved to obtain

$$
P_{0}(t)=P_{0}^{e} /\left(1+S^{0}\right) \text {. }
$$

We now write the nuclear polarization equation from Eq. (21) using the muffin-tin model in the rapid-diffusion limit (or, alternatively, using the modified shell-of-influence model with $T_{e} \ll \tau \ll T_{n}$ ). The average nuclear polarization $\bar{p}(t)$ is then given by

$$
\begin{aligned}
\frac{d \bar{p}(t)}{d t}= & -\left(\frac{1}{T_{n}^{Z}}+\frac{1}{T_{n}^{L}}\right)\left(\bar{p}-p^{e}\right)-\sum_{i=1}^{N} \xi G\left(H_{i}\right)\left\{W_{i, i+1}\left[\bar{p}-\left(P_{i}-P_{i+1}\right)\right]+W_{i, i-1}\left[\bar{p}+\left(P_{i}-P_{i-1}\right)\right]\right\} \\
& -\xi G\left(H_{0}\right)\left\{W_{0,-}\left[\bar{p}-\left(P_{0}-P_{-}\right)\right]+W_{0,+}\left[\bar{p}+\left(P_{0}-P_{+}\right)\right]\right\}-\xi G\left(H_{+}\right)\left\{W_{0,+}\left[\bar{p}-\left(P_{+}-P_{0}\right)\right]+W_{+,++}\left[\bar{p}+\left(P_{+}-P_{++}\right)\right]\right\} \\
& -\xi G\left(H_{-}\right)\left\{W_{0,-}\left[\bar{p}+\left(P_{-}-P_{0}\right)\right]+W_{-,--}\left[\bar{p}-\left(P_{-}-P_{--}\right)\right]\right\},
\end{aligned}
$$

where the subscripts $(i, i \pm 1)$ become, e.g., $(0, \pm)$ in an obvious way for the $H_{0}, H_{ \pm}$, spin packets. The prime on the summation in Eq. (37) restricts the sum to all spin packets except those at $H_{0}, H_{ \pm}$。 In Appendix B we shall deduce an estimate for $W_{i, i \pm 1}$ :

$W_{i, i \pm 1} \approx 6 \xi G\left(H_{i \pm 1}\right)\left[\frac{3}{10}\left(\frac{g_{e} \mu_{B}}{H_{0}}\right)^{2} \frac{1}{d^{3} R^{3}}\right]\left[\frac{\xi G\left(H_{i}\right)}{T_{2 e}^{B}}\right]$,

where $i$ refers here to all spin packets including those at $H_{0}, H_{ \pm}$, and $H_{ \pm \pm}$. It is useful to rewrite Eq. (38) as

$$
W_{i, i \pm 1} \approx \frac{G\left(H_{i}\right) G\left(H_{i \neq 1}\right)}{G\left(H_{00}\right) G\left(H_{ \pm}^{0}\right)} W_{\mathrm{CE}}^{\mathrm{HB}},
$$

where

$W_{\mathrm{CE}}^{\mathrm{HB}}=6 \xi G\left(H_{ \pm}^{0}\right)\left[\frac{3}{10}\left(\frac{g_{e} \mu_{B}}{H_{00}}\right)^{2} \frac{1}{d^{3} R^{3}}\right]\left(\frac{\xi G\left(H_{00}\right)}{T_{2 e}^{B}}\right)$.

Note that $W_{i, i \pm 1}=W_{i \pm 1, i}$. We use $H_{00} \approx H_{0}$ in the $\left(g_{e} \mu_{B} / H_{00}\right)^{2}$ term and define $H_{ \pm}^{0} \equiv H_{00}\left(1 \pm\left|\gamma_{n} / \gamma_{e}\right|\right)$.

When $S^{0}=0$, we have the case of nuclear spinlattice relaxation, for which Eqs. (35)-(37) can be combined to give

$$
\frac{d \bar{p}(t)}{d t}=-\frac{1}{T_{n}}\left[\bar{p}(t)-p^{e}\right],
$$

where

$$
\begin{aligned}
& \frac{1}{T_{n}}=\frac{1}{T_{n}^{\prime}}+\frac{1}{T_{n}^{C E}}, \\
& \frac{1}{T_{n}^{\prime}}=\frac{1}{T_{n}^{Z}}+\frac{1}{T_{n}^{L}},
\end{aligned}
$$

$$
\frac{1}{T_{n}^{C E}}=\sum_{i=1}^{N} \xi G\left(H_{i}\right)\left(W_{i, i+1}+W_{i, i-1}\right) .
$$

The unprimed sum is over all $N$ spin packets.

Next we consider DNP, when $S^{0}>0$. We use the enhancement $E(t) \equiv \bar{p}(t) / p^{e}$. Then Eqs. (35)(37) can be combined to give

$$
\begin{aligned}
& \frac{d E(t)}{d t}=-\frac{1}{\tau_{\mathrm{DNP}}}\left[E(t)-E_{\mathrm{ss}}\right], \\
& E_{\mathrm{ss}}=1+E_{i}\left(\frac{S^{0}}{1+S^{0}}\right)\left\{\xi\left[G\left(H_{0}\right)+G\left(H_{-}\right)\right]\left[W_{0,-} T_{n}\right]\right. \\
& \left.-\xi\left[G\left(H_{0}\right)+G\left(H_{+}\right)\right]\left[W_{0,+} T_{n}\right]\right\},(46) \\
& \tau_{\mathrm{DNP}}=T_{n},
\end{aligned}
$$

where $T_{n}$ is given in Eqs. (42)-(44). We note $E_{i}$ $=-P_{0}^{e} / p^{e}$. Using Eqs. (39), (42), and (44), $S_{\mathrm{CE}}$ $=W_{C E}^{\mathrm{IHB}} T_{n}^{\prime}$, and $G\left(H_{+}\right)+G\left(H_{-}\right) \approx 2 G\left(H_{0}\right)$, we can rewrite Eq. (46) as

$$
\begin{aligned}
E_{\mathrm{ss}}= & +E_{i}\left(\frac{S^{0}}{1+S^{0}}\right)\left(\frac{S_{\mathrm{CE}}}{1+S_{\mathrm{CE}} X}\right)\left(\frac{3\left[G\left(H_{0}\right)\right]^{2}}{G\left(H_{00}\right) G\left(H_{ \pm}^{0}\right)}\right) \\
& \times \xi\left[G\left(H_{-}\right)-G\left(H_{+}\right)\right],
\end{aligned}
$$

where the factor $X$ is defined to be $X \equiv\left(W_{\mathrm{CE}}^{\mathrm{IHB}} T_{n}^{\mathrm{CE}}\right)^{-1}$. Since $\left(T_{n}^{\mathrm{CE}}\right)^{-1}$ is a weighted average of $W_{i, i \pm 1}$ over the ESR line, whereas $W_{C E}^{\mathrm{IHB}}$ is the peak value of $W_{i, i \pm 1}$ [or close to it, depending on $G(H)$, we expect to find $X<1$. Nevertheless, because $W_{i, i \pm 1} \propto G\left(H_{i}\right)$ $\times G\left(H_{i \pm 1}\right) W_{\mathrm{CE}}^{\mathrm{IHB}}$, the contributions to $\left(T_{n}^{\mathrm{CE}}\right)^{-1}$ fall off rapidly in the wings of the ESR line, and most of the contribution comes from the center of the line where $W_{i, i \pm 1} \approx W_{\mathrm{CE}}^{\mathrm{HBB}}$. Hence we estimate $X \approx 1$. It 
is worth noting that $E_{\text {ss }}$ is a function of $N_{e}$ only when $S_{\mathrm{CE}} \lesssim 1$. For variations of $N_{e}$ such that $S_{\mathrm{CE}}$ $\gg 1, E_{\mathrm{ss}}$ is independent of $N_{e}$. Comparing Eqs. (16) and (48), we see that the microwave power dependence of $E_{\text {ss }}$ is essentially the same for both $\mathrm{CE}$ and SE in IHB ESR lines.

Let us now examine $E_{\text {ss }}$ at strong ESR saturation $\left(S^{0} \gg 1\right)$ with strong $C E\left(S_{C E} \gg 1\right)$. Then, from Eq. (48), we find

$E_{\mathrm{ss}} \approx E_{i}\left(X^{-1}\right)\left(\frac{3\left[G\left(H_{0}\right)\right]^{2}}{G\left(H_{00}\right) G\left(H_{ \pm}^{0}\right)}\right) \xi\left[G\left(H_{-}\right)-G\left(H_{+}\right)\right]$.

We note that

$$
\frac{3\left[G\left(H_{0}\right)\right]^{2}}{G\left(H_{00}\right) G\left(H_{ \pm}^{0}\right)} \lesssim 1
$$

Combining Eq. (50) with $X \approx 1$ and with $\xi\left[G\left(H_{-}\right)\right.$ $\left.-G\left(H_{+}\right)\right] \lesssim 0.1-0.01$, we see that $E_{\text {ss }}$ will be reduced from $E_{i}$ by one or more orders of magnitude. The reason for the difference between $\mathrm{SE}$ and $\mathrm{CE}$ in IHB lines is as follows: In Eq. (12), SE is pumped by the $W^{ \pm}$transitions which are at the experimenter's control and can be made much larger than $\left(T_{n}\right)^{-1}$ with sufficient ESR microwave power. However, the rates $W_{0, \pm}$ in Eq. (37), which are intrinsic to the crystal, are of the same order of magnitude as all the other rates $W_{i, i \pm 1}$, and the low values of $E_{\mathrm{ss}}$ are a consequence of the small spin-packet populations $\xi G\left(H_{0}\right), \xi G\left(H_{ \pm}\right)$. This is equivalent to the observation that $\tau_{\mathrm{DNP}} \ll T_{n}$ is possible in SE DNP, while $\tau_{\mathrm{DNP}} \approx T_{n}$ in CE DNP. We see in Eq. (49) that $E_{\mathrm{ss}} \propto\left[G\left(H_{0}\right)\right]^{2}(d G / d H)$, using a Taylor-series expansion, so that $E_{\text {ss }}$ falls off faster than $G\left(H_{0}\right)$ or $d G / d H$ in the wings of the ESR line, which provides another method of distinguishing CE from SE. The modulating $\left[G\left(H_{0}\right)\right]^{2}$ term in Eq. (49) greatly reduces $E_{\text {ss }}$ in the wings of an IHB ESR line. The reason is that in the wings the probability $\left(\xi G\left(H_{j}\right) \xi G\left(H_{k}\right) \approx\left[\xi G\left(H_{0}\right)\right]^{2}\right)$ is very small for finding two electrons $j$ and $k$ which simultaneously satisfy the CE magnetic field condition $\left(\left|H_{j}-H_{k}\right|=\left|\gamma_{n} / \gamma_{e}\right| H_{0}\right)$ and are located spatially near each other. Therefore, Eq. (38) is probably more accurate than Hill's ${ }^{23}$ expression in the wings of the ESR line, while the two treatments are similar near the center of the line. The DNP leakage factors $f_{e}^{\prime}, f_{e}^{\prime} S_{\mathrm{CE}}$ can be shown ${ }^{32}$ to reduce $E_{\mathrm{ss}}$ from Eq. (49) and possibly change the shape of the $E_{\text {ss }}$ vs $H_{0}$ curve (at fixed $\omega$ ). Their effect on $T_{n}^{\mathrm{CE}}$ is discussed in Ref。32。

Finally, it can be shown ${ }^{32}$ that $\tau_{\mathrm{DNP}} \approx T_{n}$ for all $S^{0}, f_{e}^{\prime}, f_{e}^{\prime} S_{\mathrm{CE}}$ values in the unresolved CE. The basic physics is as follows: Equation (34) shows that $\tau_{\mathrm{DNP}}$ is never shorter than $\frac{1}{2} T_{n}$ in the wellresolved $\mathrm{CE}$. In the unresolved $\mathrm{CE}$, at most only a few spin packets will contribute at a rate as fast as $2 / T_{n}$ in, e.g., Eq. (37); most of the spin packets are not driven by $W^{0}$ (directly or indirectly) and thus contribute at a rate $1 / T_{n}$. The latter dominate the net proton polarization rate, so that $\tau_{\mathrm{DNP}} \approx T_{n}$, and in no case will $\tau_{\mathrm{DNP}}$ get as short as $\frac{1}{2} T_{n}$ in the unresolved CE.

We have omitted the SE $W^{ \pm}$transitions in Secs. III A and III B in order to simplify the algebra. Our aim has been to deduce the consequences of a pure CE in order to see whether this can explain the DNP data given in II.

We now summarize some differences between this paper and Hwang and Hill's ${ }^{22,23}$ in the treatment of unresolved CE. First, they assume a strict shell-of-influence model, while we assume rapid nuclear-spin diffusion between shells. As a consequence, they predict negligible SE DNP in cases where the theory of Sec. IIC gives $E_{\text {ss }}$ peak values close to $E_{i}$ in favorable cases. Second, they use a different $W_{i, i \pm 1}$ expression from that in Eqs. (38)-(40) which, in addition to the first item, gives differing CE $E_{\text {ss }}$ results. In general, we predict much lower CE $E_{\text {ss }}$ values than they do. If $\xi$ is big enough to give a large theoretical $E_{\text {ss }}$ in, e.g., Eq. (49), this would correspond to a situation of fast spectral diffusion which would wipe out CE. Third, they do not obtain $f_{e}^{\prime}$, $f_{e}^{\prime} S_{\mathrm{CE}}$ leakage factors because of an incorrect cancellation of $\mathrm{CE}$ terms in the electron polarization equations. Fourth, they do not calculate $\tau_{\mathrm{DNP}}$. Fifth, they use a more sophisticated spin-packet line-shape function than is used here. This probably has a smaller effect on the differing CE $E_{\text {ss }}$ expressions than the first and second items above.

\section{EDDR EFFECTS ON DNP AND NUCLEAR SPIN- LATTICE RELAXATION}

The transfer of energy from one spin packet to another in an IHB ESR line, usually called spectral diffusion (or cross relaxation), has been studied often without definitive results. ${ }^{33-39}$ Let $T_{\text {SD }}^{i}$ be a characteristic time for spectral diffusion between the electrons in the spin packet at $H_{i}$ and those at $H_{i}\left(1 \pm\left|\gamma_{n} / \gamma_{e}\right|\right)$. For DNP and nuclear spin-lattice relaxation processes, the limit of a completely IHB ESR line corresponds to the case $T_{e} \ll T_{\text {SD }}^{i}$ for all spin packets $H_{i}$. Every spin packet equilibrates more rapidly with the lattice than it does with neighboring spin packets. In this case, the SE and CE theories of Secs. II and III are valid. In light of the enormous difficulties ${ }^{33}$ in estimating $T_{\mathrm{SD}}^{i}$, it is not clear how to apply the criterion $T_{e}$ $\ll T_{\text {SD }}^{i}$ in practice.

In the intermediate case, $T_{e} \approx T_{\mathrm{SD}}^{i}$, Buishvili et al. ${ }^{40}$ find that spectral diffusion in an IHB ESR line with unresolved satellites reduces the SE DNP enhancement by a factor $\left[1-\exp \left(-k \omega_{n}\right)\right]$. The term $1 / k=\left(D T_{e}\right)^{1 / 2}$, the diffusion length in frequency units, is the frequency distance over which elec- 
tron-spin energy diffuses in a time $T_{e}$, which leads to $T_{\mathrm{SD}}^{i} \approx \omega_{n}^{2} / D$. They show that the spectral diffusion coefficient $D$, and hence $k$, depend on $N_{e}$ and the spin-packet location $H_{i}$ in a complicated way.

The fast spectral-diffusion model of DNP corresponds to the limit $T_{e} \gg T_{\mathrm{SD}}^{i}$ for all spin packets $H_{i}$. For this case (and assuming unresolved satellites), Abragam and Borghini ${ }^{6,24}$ have shown that the ESR line may be treated as being essentially homogeneously broadened with EDDR serving as the main DNP mechanism. The physical idea is that the HB mechanisms are comparable to, or dominate, the IHB mechanisms, so that electronspin energy diffuses rapidly (compared to $T_{e}$ ) throughout the entire ESR line. DNP by EDDR has been discussed elsewher $\mathrm{e}^{5,6,8,24,25}$; we use the results and notation of BW here. (In particular, we use $\omega_{e}=-\gamma_{e} H_{0}$ and $\omega_{n}=-\gamma_{n} H_{0}$ in this section and in Appendix A.) We assume that all the equations in $\mathrm{BW}$ remain valid in the fast spectral-diffusion model, except that every $\omega_{L}^{2}$ in BW is replaced by $\Omega_{L}^{2}$ given by ${ }^{24}$

$$
\Omega_{L}^{2}=\omega_{L}^{2}+\left(T_{d} / T_{e}\right) \omega_{\mathrm{IHB}}^{2},
$$

where $T_{d}$ is the EDDR spin-lattice relaxation time, $\omega_{L}^{2}=\frac{1}{3} M_{2}, M_{2}$ is the HB second moment, and $\omega_{\mathrm{IHB}}^{2}$ is the second moment of the IHB frequency distribution relative to its mean frequency. Borghini ${ }^{24}$ points out that pure HB EDDR theory is not exactly the same as IHB theory with fast spectral diffusion, in particular, with regard to the microwave power dependence of the ESR saturation, which implies that the DNP leakage factors differ. We shall assume that these differences are negligible.

The enhancement $E_{\text {ss }}$ for DNP by EDDR is given in Eq. (21) in BW, which has a different microwave power dependence $S^{0}, S^{ \pm}$from that in Eqs. (14), (46), and (48) of this paper. At low microwave powers $\left(S^{0}, S^{ \pm} \ll 1\right)$, Eq. (21) in BW predicts

$$
E_{\mathrm{ss}} \propto E_{i}\left(S^{-}-S^{+}\right) \propto \frac{d G}{d H},
$$

which is actually a pure SE DNP 6 with $E_{\mathrm{ss}} \ll E_{i}$. At high powers $\left(S^{0}, S^{ \pm} \gg 1\right), E_{\mathrm{ss}}$ can be taken from
Eq. (23) of BW, replacing every $\omega_{L}^{2}$ therein by $\Omega_{L}^{2}$ :

$$
E_{\mathrm{ss}}=E_{i} \frac{\omega_{\eta} \Delta}{\Delta^{2}+\left(T_{e} / T_{d}\right) \omega_{L}^{2}+\omega_{\mathrm{IHB}}^{2}+f_{e} \omega_{n}^{2}},
$$

where $\Delta=\omega_{e}-\omega$. We find enhancement peak values $E_{\text {ss }}^{\text {peak }}$ of

$$
E_{\mathrm{ss}}^{\text {peak }}= \pm E_{i} \frac{\omega_{n}}{2\left[\left(T_{e} / T_{d}\right) \omega_{L}^{2}+\omega_{\mathrm{IHB}}^{2}+f_{e} \omega_{n}^{2}\right]^{1 / 2}}
$$

at

$$
\Delta= \pm\left[\left(T_{e} / T_{d}\right) \omega_{L}^{2}+\omega_{\mathrm{IHB}}^{2}+f_{e} \omega_{n}^{2}\right]^{1 / 2} .
$$

Since the HB contribution $\omega_{L}^{2}$ depends on $N_{e}$, while $\omega_{\text {IHB }}^{2}$ does not, two regimes are possible. For convenience, we assume $f_{e} \omega_{n}^{2} \ll\left(T_{e} / T_{d}\right) \omega_{L}^{2}+\omega_{\mathrm{IHB}}^{2}$. Then, for variations of $N_{e}$ such that $\left(T_{e} / T_{d}\right) \omega_{L}^{2}$ $\ll \omega_{\text {IHB }}^{2}$ (but spectral diffusion remains fast), we see that $E_{\mathrm{sS}}^{\text {peak }}= \pm E_{i} \omega_{n} / 2\left(\omega_{\mathrm{IHB}}^{2}\right)^{1 / 2}$ at $\Delta= \pm\left(\omega_{\mathrm{IHB}}^{2}\right)^{1 / 2}$, independent of $N_{e}$. However, if $\left(T_{e} / T_{d}\right) \omega_{L}^{2} \gtrsim \omega_{\mathrm{IHB}}^{2}$, both the peak enhancements and their field (or frequency) positions will depend on $N_{e}$. Hence, as in CE, $N_{e}$ independence of $E_{\mathrm{ss}}$ is not by itself a sufficient criterion to rule out the existence of EDDR DNP effects.

Next we examine $\tau_{\text {DNP }}$ in the EDDR DNP theory. First, we note that the measured nuclear spinlattice relaxation time $T_{n}^{m}$ can be written ${ }^{8}$

$$
\frac{1}{T_{n}^{m}}=\frac{1}{T_{n}}\left(1+\frac{S^{d}}{1+S^{d} f_{d} C_{n}}\right)
$$

where $T_{n}$ is relaxation by all modes except EDDR, and the term $\left(S^{d} / T_{n}\right) /\left(1+S^{d} f_{d} C_{n}\right)$ is nuclear relaxation through EDDR. In Secs. II and III of this paper, we have utilized only the $T_{n}$ modes of relaxation. It is easy to see that $\tau_{\mathrm{DNP}}$ in Eqs. (21c) and (22) of BW reduces to $\tau_{\mathrm{DNP}}=T_{n}^{m}$ in the lowmicrowave-power limit $\left(S^{0}, S^{ \pm} \ll 1\right)$.

Next, let us consider the high-power $\left(S^{0}, S^{ \pm}\right.$ $\gg$ 1) limit of Eqs. (21c) and (22) of BW, which can be written as

$$
\frac{1}{\tau_{\mathrm{DNP}}}=\frac{1}{T_{n}}\left(1+\frac{S^{d}}{1+S^{d} f_{d} C_{n}} F\right)
$$

where

$$
F=\frac{\left(1+S^{d} f_{d} C_{n}\right)\left(1+C_{\Delta} R\right)}{S^{d} f_{d} C_{n}+\left[S^{0}\left(1+C_{\Delta} R\right)\right] /\left\{S^{0}\left[1+\left(S^{+}+S^{-}\right) / S^{d}\right]+f_{e}\left(S^{+}+S^{-}\right)\right\}} .
$$

If $F=1$, then $\tau_{\mathrm{DNP}}=T_{n}^{m}$. There are two limits of Eqs. (56) and (57). First, if $S^{d} f_{d} C_{n} \ll 1$ and $f_{e} \ll 1$, then we find from Eq. (55)

$$
\frac{1}{T_{n}^{m}}=\frac{1}{T_{n}}\left(1+S^{d}\right)
$$

and from Eqs. (56)-(58)

$$
\frac{1}{\tau_{\mathrm{DNP}}}=\frac{1}{T_{n}^{m}}+W^{+}+W^{-} \text {. }
$$

The second limit is $S^{d} f_{d} C_{n} \gg 1$, in which case Eq. 


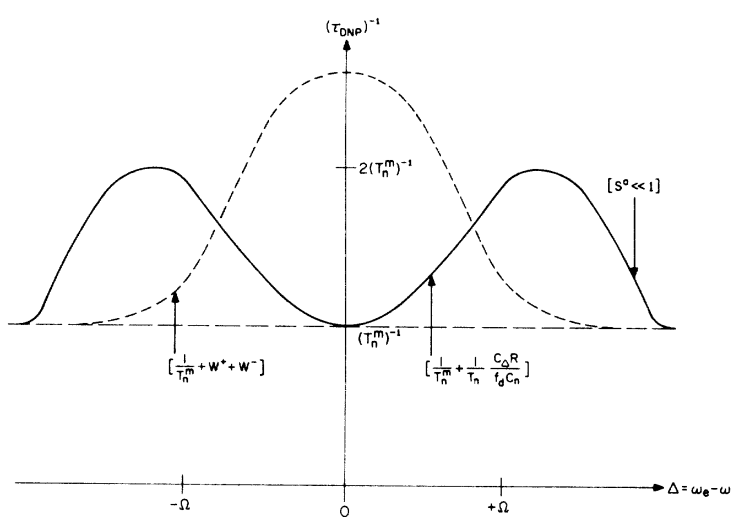

FIG. 7. Rough sketch of the DNP pump rate $\left(\tau_{\mathrm{DNP}}\right)^{-1}$ vs $\Delta$ at fixed $H_{0}$ for DNP by EDDR in the high-microwavepower limit $\left(S^{0}, S^{ \pm} \gg 1\right)$. The solid line is Eq. (61) in the text for $|\Delta|<\Omega$. In the wings of the ESR line, $S^{0} \ll 1$, so $\left(\tau_{\mathrm{DNP}}\right)^{-1}$ peaks at $|\Delta| \geqslant \Omega$ and falls off to $\left(T_{n}^{m}\right)^{-1}$ at $|\Delta|$ $\gg \Omega$. The short-dashed line is Eq. (59) in the text, assuming $\left(W^{\ddagger}\right)_{\max } \gtrsim\left(T_{n}^{m}\right)^{-1}$. The long-dashed line is $\left(\tau_{\mathrm{DNP}}\right)^{-1}$ $=\left(T_{n}^{m}\right)^{-1}$. This sketch is not based on any detailed calculations.

(55) leads to

$$
\frac{1}{T_{n}^{m}}=\frac{1}{T_{n}}\left(1+\frac{1}{f_{d} C_{n}}\right)
$$

and Eqs. (56), (57), and (60) lead to

$$
\frac{1}{\tau_{\mathrm{DNP}}}=\frac{1}{T_{n}^{m}}+\frac{1}{T_{n}}\left(\frac{C_{\Delta} R}{f_{d} C_{n}}\right)
$$

Since $1 / T_{n} f_{d} C_{n}<1 / T_{n}^{m}$, and $C_{\Delta} R=\Delta^{2} T_{d} / \Omega_{L}^{2} T_{e} \leqslant 1$ in the region where $S^{0}, S^{ \pm} \gg 1$, Eq. (61) predicts

$$
\frac{1}{T_{n}^{m}} \leq \frac{1}{\tau_{\mathrm{DNP}}} \leqslant \frac{2}{T_{n}^{m}} \text {. }
$$

Rough sketches (not based on any detailed calculations) of Eqs. (59) and (61) are given in Fig. 7. The case $S^{d} f_{d} C_{n} \approx 1$, governed by Eqs. (56) and (57), is intermediate between these two limiting cases. The solid line in Fig. 7 is Eq. (61) for $|\Delta|<\Omega$, where $\Omega=\left(T_{d} / T_{e}\right) \Omega_{L}^{2}$ and $C_{\Delta} \propto \Delta^{2}$. The solid-line rate $1 / \tau_{\text {DNP }}$ peaks at $|\Delta| \geqslant \Omega$ and falls off to $1 / T_{n}^{m}$ at $|\Delta| \gg \Omega$, since the condition $S^{0} \ll 1$ holds in the wings of the ESR line.

All calculations of EDDR ESR, $T_{n}^{m}$, and DNP effects to date have assumed an isotropic electron $g$ factor $g_{e}$, i. e. , isotropic $\gamma_{e} \cdot 5,6,8,24,25,29,30,40$ However, many experiments have been done in anisotropic crystals, e.g., in rare-earth-doped lanthanum magnesium nitrate (LMN). ${ }^{1,3,8}$ Since the experiments in this group have been done in axially symmetric $\mathrm{LMN}^{8}$ and $\mathrm{YES}^{10,16,17}$ crystals with $\theta$ (the angle between $\overrightarrow{\mathrm{H}}_{0}$ and the crystal symmetry axis) near $0^{\circ}$, we have developed the anisotropic EDDR theory only for the special case of axial symmetry with $\theta=0^{\circ}$, and with the ESR rf field $\overrightarrow{\mathrm{H}}_{1}(t)$ perpendicular to $\overrightarrow{\mathrm{H}}_{0}$. The results of these calculations (given in Appendix A) make only implicit changes in the equations in this section.

\section{THREE-SPECTRAL-REGION MODEL}

A given ESR line is usually considered to be either HB or IHB or some intermediate mixture for all its frequency (or field) components. However, there may be cases in which a wide ESR line is HB near its absorption peak and IHB in its wings. The argument for this may be illustrated with the use of the Kittel and Abrahams ${ }^{26}$ theory summarized in Appendix A.

Suppose first than an entire ESR line is predominantly IHB and that $T_{e}$ is much shorter than any $T_{2 e}$ process. Then the spin-packet width $\xi$ is given by Eq. (7) or (8) with $H_{\text {in }} \propto 1 / T_{e}$. In this case, $\xi$ is uniform throughout the ESR line, and the electrons in a given spin packet are effectively decoupled magnetically from the electrons in all the other spin packets. We may then treat the electrons in a given packet at magnetic field $H_{i}$ (Fig. 6) as an isolated group of spins having an HB magnetic dipole-dipole width $\left(T_{2 e}^{B}\right)_{i}^{-1}=\Delta_{B}^{i}$ $=5.3 f_{i} A_{B}$. If $f_{i}=\xi G\left(H_{i}\right) f$ is the fraction of spins in that spin packet, then we can rewrite this as $\left(T_{2 e}^{B}\right)_{i}^{-1}=\xi G\left(H_{i}\right) \Delta_{B}$. First, we note that the Kittel and Abrahams width $\left(T_{2 e}^{B}\right)^{-1}=\Delta_{B}$ is only an upper limit for the HB width in crystals with strong IHB mechanisms, since $\Delta_{B}^{i} / \Delta_{B}=\xi G\left(H_{i}\right) \ll 1$. Second, we see that $\left(T_{2 e}^{B}\right)_{i}^{-1}$ may vary greatly from the center of an IHB line to the wings, e.g., with an approximately Gaussian $G\left(H_{i}\right)$ as in II.

So far we have postulated $T_{e} \ll\left(T_{2 e}^{B}\right)_{i}$ for all spin packets $H_{i}$ in the ESR line. But if this does not hold, we could consider a three-spectral-region model for wide IHB ESR lines with significant HB interactions (Fig. 8). In region $I$ at and near the ESR line center, where $\left(T_{2 e}^{B}\right)_{i} \ll T_{e}$, there is essentially HB behavior or, equivalently, IHB behavior with fast spectral diffusion, resulting in

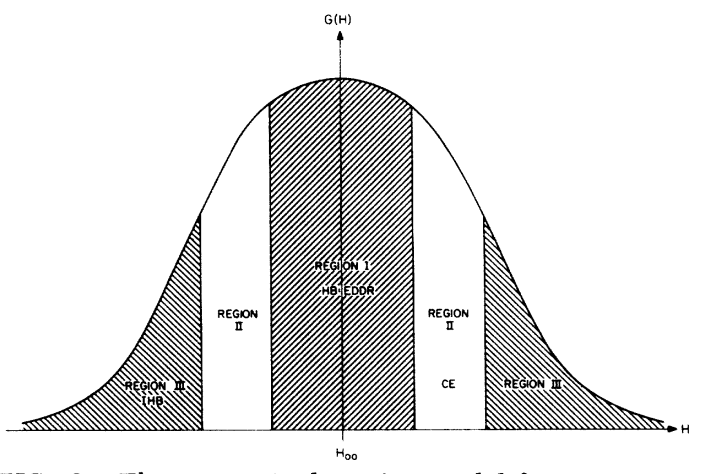

FIG. 8. Three-spectral-region model for IHB ESR lines with significant $\mathrm{HB}$ interactions in the center of the line. 
EDDR contributions to DNP and nuclear spin-lattice relaxation (Sec. IV). Region II has negligible spectral diffusion, and is thus IHB, but has sufficient numbers of electrons in the spin packets so that $W_{i, i \pm 1}$ is large. We denote this region CE (Sec. III), since CE cannot occur in region I and is negligible in region III where $W_{i, i \pm 1}$ is very small. In region II, no EDDR effects occur, but $T_{n}^{Z}$ and SE DNP processes will compete with CE. In region III, one has $T_{e} \ll\left(T_{2 e}^{B}\right)_{i}$, and the ESR, DNP, and nuclear relaxation behavior is essentially IHB (Sec. II) with no CE. The basic physical model is that the IHB mechanisms put different electrons at different Larmor frequencies. In region III, it is highly improbable that an electron will find another electron which both is on "speaking terms" with it (i.e., $S_{\neq}^{i} S_{\mp}^{j}$ transitions are energetically possible) and is spatially nearby. At the center of the line in region $\mathrm{I}$, it becomes relatively more probable to find two electrons near each other and on "speaking terms." If the transition from negligible spectral diffusion to fast spectral diffusion in the ESR line is very sharp, then the CE region II would be wiped out. Data suggesting this is given in II. Nevertheless, we shall retain all three regions for generality. This crude three-spectral-region model has rectangular regions and ignores overlap between them. It has not been derived from first principles, but rather is intended to be suggestive of the complexities of DNP and $T_{n}^{m}$ behavior in some crystals. Problems of nuclear magnetization spatial homogeneity, correlations between electron spectral and spatial positions, and electron jumps between spin packets have been discussed elsewhere. ${ }^{32}$

\section{SUMMARY}

We have derived theoretical expressions for DNP and nuclear spin-lattice relaxation in diamagnetic dielectric crystals diluted with electron paramagnetic impurities. The steady-state DNP enhancement $E_{\text {ss }}$ and the DNP pump time $\tau_{\text {DNP }}$ have been calculated for SE, CE, and EDDR DNP, assuming (i) a predominantly IHB ESR line with uniform spin-packet widths (for SE and CE, but not EDDR DNP), (ii) rapid nuclear-spin diffusion, (iii) the electron and nuclear high-temperature limit, (iv) no phonon bottleneck, and (v) isotropic electron impurities or axially symmetric electrons whose symmetry axis ( $c$ axis) is parallel to $\overrightarrow{\mathrm{H}}_{0}$. DNP results for IHB SE, which include DNP leakage factors, predict $E_{\text {ss }}$ comparable to the ideal enhancement $E_{i}$ in favorable cases, with $E_{\mathrm{ss}}$ vs $H_{0}$ curves not proportional to the ESR lineshape derivative at high microwave powers, contrary to early theories of IHB SE. The effects of electron jumps between spin packets have been discussed. DNP and $T_{n}$ have been calculated for the cross effect for both the well-resolved and unresolved limits. We get larger SE DNP and smaller CE DNP for the unresolved case than do Hwang and Hill, and consider CE DNP leakage factors they omitted. The inequality $T_{n} / 2 \leq \tau_{\mathrm{DNP}} \leq T_{n}$ is found for the well-resolved CE, while $\tau_{\mathrm{DNP}} \approx T_{n}$ is estimated for the unresolved $C E$, different from the general SE case where $\tau_{\mathrm{DNP}} \ll T_{n}$ often occurs. DNP by EDDR has been extended from the pure HB ESR limit to the IHB case with fast spectral diffusion, as suggested by Abragam and Borghini, ${ }^{8,24}$ and was further extended from electronic isotropy to axially symmetric electrons with the $c$ axis parallel to $\overrightarrow{\mathrm{H}}_{0}$. Various limits of $E_{\mathbf{s s}}$ and $\tau_{\text {DNP }}$ have been compared and contrasted for SE, $\mathrm{CE}$, and EDDR DNP to enable experimenters to differentiate these three DNP mechanisms, if possible. We propose a three-spectral-region model for wide ESR lines, which may exhibit IHB behavior in the wings of the line, HB EDDR effects near the ESR absorption peak, and CE effects in between. This theoretical study has been motivated by the DNP and $T_{n}^{m}$ data in YES : Er to be presented in II.

\section{ACKNOWLEDGMENTS}

I would like to thank C. D. Jeffries, J. P. Wolfe, H. J. Stapleton, M. Borghini, and J. Jeener for helpful conversations, and D. A. Hill for a copy of his Ph. D. dissertation.

\section{APPENDIX A: EDDR THEORY FOR AN AXIALLY SYMMETRIC ELECTRON SYSTEM AT $\theta=0^{\circ}$}

An axially symmetric crystal is described by the two electron $g$-value tensor components $g_{\|}$and $g_{\perp}$ and by the resonance condition $\hbar \omega=g_{e} \mu_{B} H_{0}$ at angle $\theta$, where ${ }^{5}$

$$
g_{e}=\left(g_{\|}^{2} \cos ^{2} \theta+g_{\perp}^{2} \sin ^{2} \theta\right)^{1 / 2} .
$$

The problem in the anisotropic EDDR case is handling the $W^{d}$ and $\omega_{L}^{2}$ terms. ${ }^{8}$ For $\theta=0^{\circ}$, the truncated electron dipole-dipole Hamiltonian $H_{S S}^{0}$ may be written in analogy with the isotropic case $^{25,41,42}$

$$
H_{S S}^{0}=\frac{\mu_{B}^{2}}{2} \sum_{i \neq j=1}^{N_{e}} r_{i j}^{-3}\left(A_{i j}+B_{i j}\right)\left(1-3 \cos ^{2} \theta_{i j}\right),
$$

where

$$
\begin{aligned}
& A_{i j}=g_{\Perp}^{2} S_{z}^{i} S_{z}^{j}, \\
& B_{i j}=-\frac{1}{4} g_{\perp}^{2}\left(S_{+}^{i} S_{-}^{j}+S_{-}^{i} S_{+}^{j}\right) .
\end{aligned}
$$

$B_{i j}$ is easily transformed to ${ }^{42}$

$$
B_{i j}=\frac{1}{2} g_{\perp}^{2}\left(S_{z}^{i} S_{z}^{j}-\vec{S}_{i} \cdot \vec{S}_{j}\right) \text {. }
$$

Inspection shows that the formulation of the moment method of linewidth calculations for the iso- 
tropic $g_{e}$ case $^{42}$ does not change when we go to axial symmetry at $\theta=0^{\circ}$. The second moment $M_{2}$ (in units of $\omega$ ) is ${ }^{42}$

$$
M_{2}=\frac{\operatorname{Tr}\left(\left[H_{S S}^{0}, S_{x}\right]^{2}\right)}{\left(\hbar^{2}\right) \operatorname{Tr}\left(S_{x}^{2}\right)}
$$

Since the $S_{x}=\sum_{i=1}^{N_{e}} S_{x}^{i}$ terms commute with the $\overrightarrow{\mathrm{S}}_{i} \cdot \overrightarrow{\mathrm{S}}_{j}$ terms in Eq. (A6), we see that

$$
M_{2} \propto\left(g_{\|}^{2}+\frac{1}{2} g_{1}^{2}\right)^{2} .
$$

The $g_{\|}^{2}$ term comes from the $A_{i j}$ static IHB broadening, while the $g_{\perp}^{2}$ term originates in the dynamic HB broadening $B_{i j}$.

Kittel and Abrahams ${ }^{26,42}$ have calculated linewidths due to the magnetic dipole-dipole interaction in paramagnetically dilute crystals with isotropic $g_{e}$, assuming completely HB lines, and explicitly ignoring exchange, hyperfine, and all IHB mechanisms. Let $f$ be the fraction of magnetic sites occupied by the dilute impurities. Using a moment calculation, they show that the ESR line shape is roughly Gaussian for $f>0,10$, and approximates a cutoff Lorentzian for $f<0.01$. In the latter case they find for a simple cubic lattice with $\overrightarrow{\mathrm{H}}_{0}$ along the 100 axis that the Lorentzian halfwidth at half-maximum $\Delta_{1 / 2}$ (in units of $\omega$ ) is given by $\Delta_{1 / 2}=5.3 f A$, where $A=\left(g_{e} \mu_{B}\right)^{2} / \hbar a^{3}$. The cubic lattice parameter $a$ relates the number of electron impurities $/ \mathrm{cm}^{3} N_{e}$ to $f$ by $N_{e}=f / a^{3}$. We will use $T_{2 e}=1 / \Delta_{1 / 2}$. They find that the Lorentzian cutoff frequency $\alpha$ (in units of $\omega$ ) is $\alpha=2.2 \mathrm{~A}$. In a crystal which is not cubic along the 100 axis, we expect results similar to the above but with numerical factors differing from Kittel and Abrahams's parameters by factors of order unity. For convenience, we use their factors $5.3(2.2)$ in $\Delta_{1 / 2}(\alpha)$ below.

We now extend the Kittel and Abrahams calculation to an anisotropic ESR line with axial symmetry at $\theta=0^{\circ}$. Equations (A2)-(A7) suggest that for $f<0.01$, we still have a Lorentzian line shape with

$$
\Delta_{1 / 2}=5.3 f A^{\prime},
$$

where

$$
A^{\prime}=\frac{2}{3}\left(g_{\| 1}^{2}+\frac{1}{2} g_{1}^{2}\right)\left(\mu_{B}^{2} / \hbar a^{3}\right) \text {. }
$$

When $A^{\prime}$ is expressed in magnetic field units at $\theta=0^{\circ}$, the $\mu_{B}^{2} / \hbar a^{3}$ term in Eq. (A9) is replaced by $\mu_{B} / g_{\| \prime} a^{3}$, and similarly for the related terms $A_{A}$ and $A_{B}$ below. For noncubic crystals $a^{3}$ is defined by $a^{3}=f / N_{e}$. We now separate the $A_{i j}$ and $B_{i j}$ terms in $\Delta_{1 / 2},\left(T_{2 e}\right)^{-1}$, and $\alpha$. We define $\Delta_{A}$ $=\left(T_{2 e}^{A}\right)^{-1}=5.3 f A_{A}$ and $\Delta_{B}=\left(T_{2 e}^{B}\right)^{-1}=5.3 f A_{B}$, where $A_{A}=2\left(g_{\|} \mu_{B}\right)^{2} / 3 \hbar a^{3}$ and $A_{B}=\left(g_{\perp} \mu_{B}\right)^{2} / 3 \hbar a^{3}$. We also use $\alpha_{A}=2.2 A_{A}$ and $\alpha_{B}=2.2 A_{B}$. Thus we can write

$$
\begin{aligned}
& \Delta_{1 / 2}=\Delta_{A}+\Delta_{B}, \\
& \frac{1}{T_{2 e}}=\frac{1}{T_{2 e}^{A}}+\frac{1}{T_{2 e}^{B}}, \\
& \alpha=\alpha_{A}+\alpha_{B} .
\end{aligned}
$$

We now discuss DNP and $T_{n}^{m}$ in an axially symmetric electron system at $\theta=0^{\circ}$. All the equations in BW and in Sec. IV of this paper remain valid except for the changes indicated in this paragraph. In most equations, $g_{e}$ becomes $g_{11}$ and $\gamma_{e}$ becomes $\gamma_{11}$, except as noted, where $g_{11} \mu_{B}=\left|\gamma_{\|}\right| \hbar$. Thus, we have $\omega_{e}=-\gamma_{11} H_{0}, \Delta$ remains $\Delta=\omega_{e}-\omega$, and the SE satellites occur at magnetic fields $H_{ \pm}$ $=H_{0}\left[1_{ \pm}\left|\gamma_{n} / \gamma_{11}\right|\right]$ at fixed $\omega$. Jeffries ${ }^{43}$ has given general expressions for $\omega_{1}^{2}$ and $\epsilon^{2} / \omega_{n}^{2}$ for general axial symmetry. For $\theta=0^{\circ}$, and for $\overrightarrow{\mathrm{H}}_{1}(t)$ perpendicular to $\overrightarrow{\mathrm{H}}_{0}$, they reduce to ${ }^{43}$

$$
\begin{aligned}
& \omega_{1}^{2}=\left(\gamma_{\perp} H_{1}\right)^{2}=\left(g_{\perp} \mu_{B} H_{1} / \hbar\right)^{2}, \\
& \epsilon^{2}=3\left(\gamma_{n} \gamma_{\| I} \hbar\right)^{2} / 10 d^{3} R^{3},
\end{aligned}
$$

and

$$
\epsilon^{2} / \omega_{n}^{2}=\frac{3}{10}\left(\frac{g_{11} \mu_{B}}{H_{0}}\right)^{2} \frac{1}{d^{3} R^{3}},
$$

which appear in Eqs. (1), (4)-(7), and (9)-(11) of BW. We use $g_{\perp} \mu_{B}=\left|\gamma_{\perp}\right| \hbar$. For the limit $\left(\omega_{n} T_{2 e}^{B}\right)^{2}$ $\gg 1$, Eq. (11) of BW becomes

$$
W^{d}=\frac{3}{10}\left(\frac{g_{\|} \mu_{B}}{H_{0}}\right)^{2} \frac{1}{d^{3} R^{3}} \frac{1}{T_{2 e}^{B}},
$$

since it is only the $B_{i j}$ terms in $H_{S S}^{0}$ which cause the $W^{d} \propto\left(T_{2 e}^{B}\right)^{-1}$ forbidden electron spin-spin transitions $\left(\Delta M_{S}^{i}= \pm 1, \Delta M_{S}^{j}=\mp 1, \Delta M_{I}= \pm 1\right)$. (These are the same transitions as in CE.) Then Eq. (12) of BW becomes $S^{d}=W^{d} T_{n}=T_{e} / T_{2 e}^{B}$. The distinction between $T_{2 e}^{B}$ and $T_{2 e}$ corresponds to a factor $\approx 3$ for $W^{d}$ in the isotropic case [Eq. (11) of $\mathrm{BW}]$ and thus was not emphasized in BW. For the anisotropic case, where $g_{\|}$and $g_{\perp}$ may differ greatly, it becomes important (see II). Thus $g\left(\omega_{n}\right)$ is wrong in Eq. (6) of BW. The relation $\omega_{L}^{2}$ $=\frac{1}{3} M_{2}$ still holds, but Eqs. (A2) $-(\mathrm{A} 7)$ are needed here to calculate $M_{2}$. Since the $A_{i j}\left(B_{i j}\right)$ terms in $H_{S S}^{0}$ are IHB (HB) mechanisms, an ESR line at $\theta=0^{\circ}$ broadened predominantly by $H_{S S}^{0}$ is HB when $g_{\perp} \gtrsim g_{\| 1}$, but becomes IHB when $g_{\perp} \ll g_{\|}$. Finally, $g_{e}$ becomes $g_{\|}$in Eqs. (19a) and (19b) of BW, and Eq. (20) of BW remains valid with $H_{L}^{2}=\omega_{L}^{2} /\left(\gamma_{11}\right)^{2}$.

It can be shown ${ }^{32}$ that the Kittel and Abrahams cutoff frequency $\alpha_{B}$ has two effects on nuclear relaxation and DNP which were not discussed in BW. First, the Lorentzian spectrum $\left(\propto T_{2 e}^{B} /[1\right.$

$\left.\left.+\left(\omega_{n} T_{2 e}^{B}\right)^{2}\right]\right)$ usually used for $W^{d}$ [see Eqs. (9)-(11) in BW], which reduces to Eq. (A16) when $\left(\omega_{n} T_{2 e}^{B}\right)^{2}$ $\gg 1$, is only valid if $\omega_{n} \leq \alpha_{B}$. In the Kittel and Abrahams picture for an HB ESR line, we have 
$W^{d}=0$ if $\omega_{n}>\alpha_{B}$. Second, if $\omega_{n} \geq \alpha_{B}$, the shell-ofinfluence model breaks down; this model remains valid for EDDR and CE theories of DNP and $T_{n}^{m}$ only if $\omega_{n} \ll \alpha_{B}$.

\section{APPENDIX B: DERIVATION OF EQUATION (38)}

We use the results of the Kittel and Abrahams theory (Appendix A) and of Sec. V to derive Eq. (38) in Sec. III B. We extend the well-resolved $\mathrm{CE}$ result [Eq. (23)] to an IHB line with unresolved satellites. For the spin packet at $H_{i}$, the effective $\left(T_{2 e}^{B}\right)_{i}$ which should replace $T_{2 e}$ in Eq. (23) is, from Sec. V,

$$
\left(T_{2 e}^{B}\right)_{i}^{-1}=\xi G\left(H_{i}\right)\left(T_{2 e}^{B}\right)^{-1},
$$

where $\left(T_{2 \varepsilon}^{B}\right)^{-1}$ is the HB width that the ESR line would have if no IHB mechanisms existed in the crystal. That is, only the fraction $\xi G\left(H_{i}\right)$ of the electrons are on "speaking terms" for allowed electron spin-spin transitions $\left(\Delta M_{S}^{j}= \pm 1, \Delta M_{S}^{k}\right.$ $=\mp 1)$ in the $H_{i}$ spin packet. The $\left(T_{2 e}^{B}\right)_{i}$ of Eq. (B1) determines the Lorentzian spectral-distribution function that drives the nuclear CE transitions.

There is a second factor that is spatial. Consider a given electron which is in the $H_{i}$ spin packet. For a uniform electron distribution, there are $\approx 6$ sites at a distance $\approx 2 R$ from the electron, each of which has a probability $\xi G\left(H_{i \pm 1}\right)$ of occupying the $H_{i \pm 1}$ spin packet, necessary for $\mathrm{CE}$ to occur. Hence, for a given electron at $H_{i}$, Eq. (23) must be generalized in the IHB case to

$W_{i, i \pm 1} \approx 6 \xi G\left(H_{i \pm 1}\right)\left[\frac{3}{10}\left(\frac{g_{e} \mu_{B}}{H_{0}}\right)^{2} \frac{1}{d^{3} R^{3}}\right]\left(\frac{\xi G\left(H_{i}\right)}{T_{2 e}^{B}}\right)$,

which is the same as Eq. (38). Equation (B2) is only an estimate, but may be moderately reliable in the wings of an IHB ESR line. Near the center of the line, spectral diffusion may wipe out CE or at least break down the idea of spin packets of uniform width assumed here.
*Present address: U. S. Arms Control and Disarmament Agency, Washington, D. C. 20451.

${ }^{1}$ O. S. Leifson and C. D. Jeffries, Phys. Rev. $\underline{122}$, 1781 (1961).

${ }^{2}$ C. D. Jeffries, Dynamic Nuclear Orientation (Interscience, New York, 1963).

${ }^{3}$ T. J. Schmugge and C. D. Jeffries, Phys. Rev. 138, A1785 (1965).

${ }^{4}$ C. D. Jeffries, Technical Report No. UCB-34P20-T-1, Physics Department, University of California, 1966 (unpublished).

${ }^{5}$ C. D. Jeffries, in Electron Paramagnetic Resonance, edited by S. Geschwind (Plenum, New York, 1972), pp. 217-262.

${ }^{6} \mathrm{~A}$. Abragam and M. Borghini, in Progress in Low Temperature Physics, edited by C. J. Gorter (NorthHolland, Amsterdam, 1964), Vol. 4, pp. 384-449.

${ }^{7}$ M. Borghini, Phys. Rev. Lett. 16, 318 (1966).

${ }^{8}$ C. E. Byvik and D. S. Wollan, Phys. Rev. B 10,791 (1974), denoted BW, hereafter.

${ }^{9}$ A. M. Portis, Phys. Rev. 91, 1071 (1953).

${ }^{10}$ D. S. Wollan, following paper, Phys. Rev. B $\underline{13}, 3686$ (1976), denoted II, hereafter.

${ }^{11}$ J. L. Motchane, Ann. Phys. (Paris) 7, 139 (1962).

${ }^{12}$ M. Abraham, M. A. H. McCausland, and F. N. H. Robinson, Phys. Rev. Lett. 2, 449 (1959).

${ }^{13}$ G. R. Khutsishvili, Usp. Fiz. Nauk 87, 211 (1965) [Sov. Phys. -Usp. 8 , 743 (1966)].

${ }^{14} \mathrm{P}$. Zegers and R. van Steenwinkel, Physica (Utr.) 33 , 332 (1967).

${ }^{15}$ Khutsishvili is not clear about the second mechanism in Ref. 13. Since he uses a single shell-of-influence spin-diffusion calculation, we have interpreted his model to include electron jumps between spin packets.

${ }^{16}$ D. S. Wollan and J. W. Poulton, Phys. Lett. A $\underline{33}, 33$ (1970).

${ }^{17} \mathrm{D}$. S. Wollan, in Proceedings of the Second International Conference on Polarized Targets, Lawrence Berkeley Laboratory Report LBL-500, UC-34, TID-
4500, 1971, pp. 43-46 (unpublished).

${ }^{18}$ A. V. Kessenikh, V. I. Lushchikov, A. A. Manenkov, and Y. V. Taran, Fiz. Tverd. Tela 5, 443 (1963) [Sov. Phys. -Solid State $\underline{5}, 321$ (1963)].

${ }^{19}$ A. V. Kessenikh and A. A. Manenkov, Fiz. Tverd. Tela 5, 1143 (1963) [Sov. Phys. -Solid State 5, 835 (1963)].

${ }^{20}$ A. V. Kessenikh, A. A. Manenkov, and G. I. Pyatnitskii, Fiz. Tverd. Tela $\underline{6}, 827$ (1964) [Sov. Phys.-Solid State 6 , 641 (1964)].

${ }^{21}$ C. F. Hwang and D. A. Hill, Phys. Rev. Lett. $\underline{18}$, 110 (1967).

${ }^{22}$ C. F. Hwang and D. A. Hill, Phys. Rev. Lett. 19 , 1011 (1967).

${ }^{23}$ D. A. Hill, Ph. D. dissertation (Northwestern University, Evanston, 1970) (unpublished).

${ }^{24} \mathrm{M}$. Borghini, in Ref. 17 , pp. 1-32.

${ }^{25} \mathrm{M}$. Goldman, Spin Temperature and Nuclear Magnetic Resonance in Solids (Oxford U. P. , Oxford, England, 1970).

${ }^{26}$ C. Kittel and E. Abrahams, Phys. Rev. 90, 238 (1953).

${ }^{27}$ W. E. Blumberg, Phys. Rev. 119, $79(1 \overline{960})$.

${ }^{28}$ I. J. Lowe and D. Tse, Phys. Rev. 166, 279 (1968).

${ }^{29}$ G. R. Khutsishvili, Usp. Fiz. Nauk $\underline{96}, 441$ (1968) [Sov. Phys. -Usp. 11, 802 (1969)].

${ }^{30}$ G. R. Khutsishvili, in Ref. 6, Vol. 6, pp. 375-404.

${ }^{31} \mathrm{~A}$ simple adiabatic calorimetric problem illustrates this point. Consider $m$ "hot" metal cubes at $80^{\circ} \mathrm{C}$ and $n$ "cold" metal cubes at $40^{\circ} \mathrm{C}$. All cubes have the same temperature-independent heat capacity. If the cubes are brought together, the final equilibrium temperature of the $m+n$ cubes is $T=60^{\circ}+20^{\circ}[(m-n) /(m+n)]$.

${ }^{32} \mathrm{D}$. S. Wollan (unpublished), available upon request.

${ }^{33}$ W. B. Mims, in Ref. 5, pp. 263-351.

${ }^{34}$ A. M. Portis, Phys. Rev. 104, 584 (1956).

${ }^{35} \mathrm{~N}$. Bloembergen, S. Shapiro, P. S. Pershan, and J.

O. Artman, Phys. Rev. 114, 445 (1959).

${ }^{36}$ A. Kiel, Phys. Rev. 120,137 (1960).

${ }^{37}$ A. Kiel, Phys. Rev. $\underline{125}, 1451$ (1962). 
${ }^{38}$ J. R. Klauder and P. W. Anderson, Phys. Rev. 125, 912 (1962).

${ }^{39}$ W. B. Mims, K. Nassau, and J. D. McGee, Phys.

Rev. 123, 2059 (1961).

${ }^{40}$ L. L. Buishvili, M. D. Zviadadze, and G. R. Khutsishvili, Zh. Eksp. Teor. Fiz. 54, 876 (1968) [Sov. Phys.-
JETP 27, 469 (1968)].

${ }^{41}$ J. H. Van Vleck, Phys. Rev. 74, 1168 (1948).

${ }^{42} \mathrm{~A}$. Abragam, The Principles of Nuclear Magnetism (Oxford U.P., Oxford, England, 1961), Chap. 4.

${ }^{43}$ Reference 5, Eqs. (23a) and (23b). 